\title{
Identification of a competing endogenous RNA network related to immune signature in clear cell renal cell carcinoma
}

\author{
Yuke Zhang ${ }^{1}$, Jiangwen Dai ${ }^{2}$, Weifeng Huang ${ }^{3}$, Qingsong Chen ${ }^{4}$, Wei Chen ${ }^{5}$, Qiying $\mathrm{He}^{1}$, \\ Feng Chen ${ }^{6}$, Peng Zhang ${ }^{1}$ \\ ${ }^{1}$ Department of Urology, West China Hospital of Sichuan University, Chengdu, China \\ ${ }^{2}$ Department of Oncology, Chengdu Fifth People's Hospital of Chengdu University of TCM, Chengdu, China \\ ${ }^{3}$ Department of Hepatobiliary Surgery, The First Affiliated Hospital of Chongqing Medical University, Chongqing, \\ China \\ ${ }^{4}$ Department of Traumatology, Chongqing University Central Hospital, Chongqing, China \\ ${ }^{5}$ Department of Hepatobiliary Surgery, Daping Hospital, Army Medical University, Chongqing, China \\ ${ }^{6}$ Department of Integrated Care Management Center, West China Hospital of Sichuan University, Chengdu, China
}

Correspondence to: Feng Chen, Peng Zhang; email: chenfeng2538@163.com, https://orcid.org/0000-0002-6534-9436; zhangpenghuaxi@126.com, https://orcid.org/0000-0003-1702-7157

Keywords: clear cell renal cell carcinoma, competing endogenous RNA, tumor microenvironment, prognosis, immune cell infiltration

Received: July 23, 2021

Accepted: December 8, 2021

Published: December 27, 2021

Copyright: (c) 2021 Zhang et al. This is an open access article distributed under the terms of the Creative Commons Attribution License (CC BY 3.0), which permits unrestricted use, distribution, and reproduction in any medium, provided the original author and source are credited.

\section{ABSTRACT}

Clear cell renal cell carcinoma (ccRCC) is a fatal cancer of the urinary system. Long non-coding RNAs (IncRNAs) act as competitive endogenous RNAs (ceRNAs) involving the cCRCC progression. However, the relationship between the ceRNA network and immune signature is largely unknown. In this study, the ccRCC-related gene expression profiles retrieved from the TCGA database were used first to identify the differentially expressed genes through differential gene expression analysis and weighted gene co-expression network analysis. The interaction among differentially expressed IncRNAs, miRNAs, and mRNAs were matched using public databases. As a result, a ceRNA network was developed that contained 144 IncRNAs, 23 miRNAs, as well as 62 mRNAs. Four of 144 IncRNAs including LINC00943, SRD5A3-AS1, LINC02345, and U62317.3 were identified through LASSO regression and Cox regression analyses, and were used to create a prognostic risk model. Then, the ccRCC samples were divided into the high- and low-risk groups depending on their risk scores. ROC curves, Kaplan-Meier survival analysis, and the survival risk plots indicated that the predictive performance of our developed risk model was accurate. Moreover, the CIBERSORT algorithm was used to measure the infiltration levels of immune cells in the ccRCC samples. The further genomic analysis illustrated a positive correlation between most immune checkpoint blockade-related genes and the risk score. In conclusion, the present findings effectually contribute to the comprehensive understanding of the ccRCC pathogenesis, and may offer a reference for developing novel therapeutic and prognostic biomarkers.

\section{INTRODUCTION}

Renal cell carcinoma (RCC) has been known to be among the most fatal cancers in the urinary system [1]. RCC consists of three major histological subtypes, including chromophobe RCC, papillary RCC, and clear cell renal cell carcinoma (ccRCC).
ccRCC is reported to be responsible for $\sim 80 \%$ of total RCC incidence [2, 3]. Surgical resection is the principal treatment strategy, as ccRCC patients are insensitive to chemotherapy and radiotherapy. However, a third of the patients who undergo surgery have a recurrence, further leading to poor prognosis and high mortality [4]. Although in recent years, 
several targeted therapeutics such as sorafenib, sunitinib, and everolimus have successfully obtained approval for the clinical application in ccRCC treatment, their treatment effects are variable and the patients' conditions usually deteriorate, which necessitates further investigation for identifying new therapeutic agents as well as prognostic biomarkers [5, 6].

Long non-coding RNAs (lncRNAs) are non-coding RNAs (ncRNAs) with a length of $>200$ nucleotides, and exert fundamental contributions to a variety of biological processes, such as chromatin organization, transcriptional regulation, and posttranscriptional modification [7]. The highly conserved small ncRNAs known as microRNAs (miRNAs) have the length of 20-25 nucleotides [8]. The competitive endogenous RNA (ceRNA) concept describes the function of lncRNAs, messenger RNAs (mRNAs), and other RNA transcript types as natural miRNA "sponges" that suppress miRNA functions via miRNA response elements (MREs) [9]. IncRNAs can reduce the affinity between miRNAs and mRNAs by competing for miRNA binding, causing aberrant expressions of mRNAs. Several studies have validated the ceRNA hypothesis, and it is widely recognized as tightly associated with the initiation and progression of various cancers $[10,11]$.

As an intricate and complex ecosystem, the tumor microenvironment (TME) comprises a variety of innate and adaptive immune cells along with the cancer cells and the surrounding stroma $[12,13]$. The infiltration of immune cells contributes to various aspects of tumor progression and is closely related to clinical outcomes [14]. For instance, the infiltration of CD8+ T cells indicates a better prognosis and response to immunotherapy in many cancers [15]. However, persistent antigen exposure reduces the propagation and cytotoxicity of $\mathrm{CD} 8+\mathrm{T}$ cells, leading to an 'exhaustion' phenotype. The exhausted state is accompanied by the enhanced expression levels of immune checkpoint blockade (ICB)-related genes, including PD-1, TIM3, and CTLA4. Blockade of these molecules can rescue the exhaustion phenotype [16]. Macrophages are a major component of immune cell types in TME. They can be activated and polarized into two diverse subtypes, the M1 and M2 macrophages, through differential stimulation by cytokines and chemokines [17]. M1 macrophages are characterized by their ability to release abundant proinflammatory cytokines, efficient antigen presentation, and tumoricidal activity [18]. On the contrary, M2 macrophages inhibit inflammatory responses and create an immunosuppressive microenvironment for tumor growth, metastasis, and angiogenesis [19]. Taken together, a better understanding of infiltration of the immune cells is a substantial step toward the diagnosis and treatment of ccRCC.

In the present study, the gene expression profiles were retrieved from The Cancer Genome Atlas (TCGA) database. The obtained data were then evaluated by differential gene expression analysis as well as weighted gene co-expression network analysis (WGCNA). Then, public databases were applied for examining the interactions among lncRNAs, miRNAs, and mRNAs, and constructing a ceRNA network. The genes within the ceRNA network were then analyzed by gene ontology (GO) and Kyoto Encyclopedia of Genes and Genomes (KEGG) pathway analyses, and the potential pathogenesis mechanisms of ccRCC were identified. Consequently, four lncRNAs were utilized to create a prognostic risk model with a validated good predictive accuracy. The ccRCC samples were graded into the high- and low-risk groups. The high-risk grouprelated genes were identified, and underwent functional enrichment analysis. Finally, the infiltration levels of immune cells and the expression levels of ICB-related genes were measured, and compared in different risk groups. In summary, our study is envisaged to produce novel insights about the carcinogenesis mechanisms of ccRCC, and provide a reliable reference for developing therapeutic targets for it.

\section{RESULTS}

\section{Differential gene expression analysis}

In order to detect the differentially expressed lncRNAs, miRNAs, and mRNAs, the TCGA database was searched for the RNA sequencing data of ccRCC samples. A total of 2653 differentially expressed IncRNAs (2040 up-regulated and 613 downregulated), 316 differentially expressed miRNAs (202 up-regulated and 114 down-regulated), and 4971 differentially expressed mRNAs (3055 up-regulated and 1916 down-regulated) were identified using the set threshold of $\mid \log 2 \mathrm{FC}$ (fold change) $\mid>1$ and false discovery rate $($ FDR $)<0.05$. The Volcano plots and heatmaps were performed to visualize and compare the distribution of the differentially expressed lncRNAs/mRNAs (Figure 1A, 1C) and differentially expressed miRNAs (Figure 1B, 1D) between ccRCC and normal renal samples.

\section{Construction of the weighted gene co-expression network for IncRNAs and mRNAs}

Functional gene clusters in ccRCC samples were determined using the WGCNA package. With a soft- 
threshold $\beta$ set at 11 , a scale-free network was obtained with the scale-free $\mathrm{R}^{2}=0.86$ (Figure 2A, 2B). Then, 12 modules were detected after merging the highly similar co-expression modules (Figure 2C). Among them, the highest positive correlation with ccRCC samples belonged to the pink module, with the module membership (MM) and gene significance (GS) correlation of 0.66 (Figure 2D, 2E). Finally, 359
lncRNAs and 1499 mRNAs were extracted from the pink module for further analysis.

\section{Construction of the weighted gene co-expression network for miRNAs}

The weighted co-expression network was developed for the miRNAs using the same method as described for
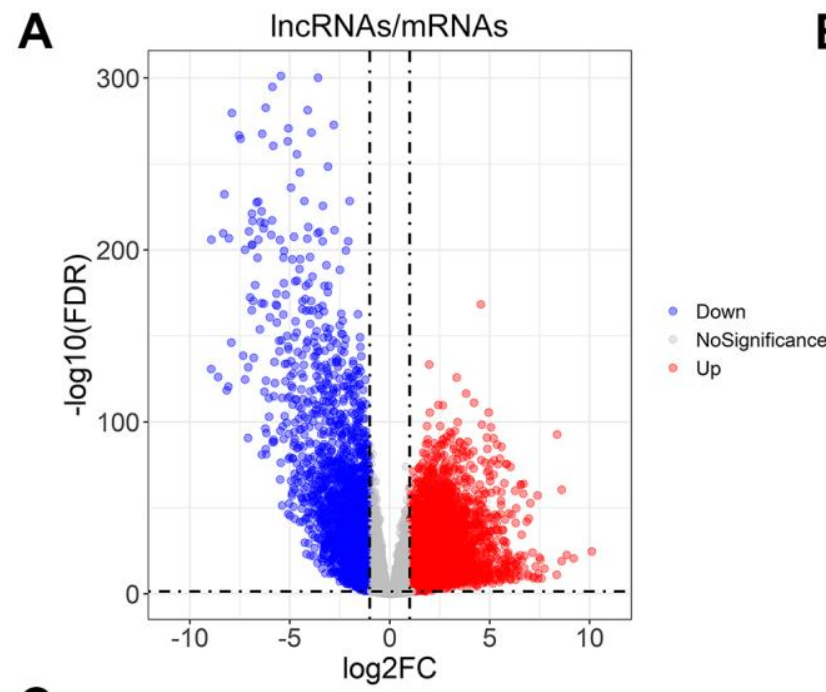

B

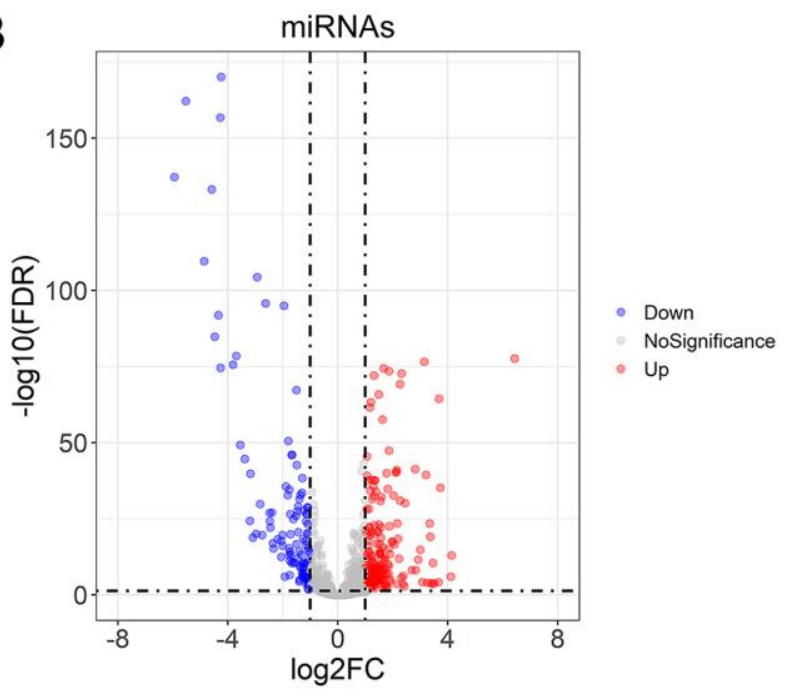

C

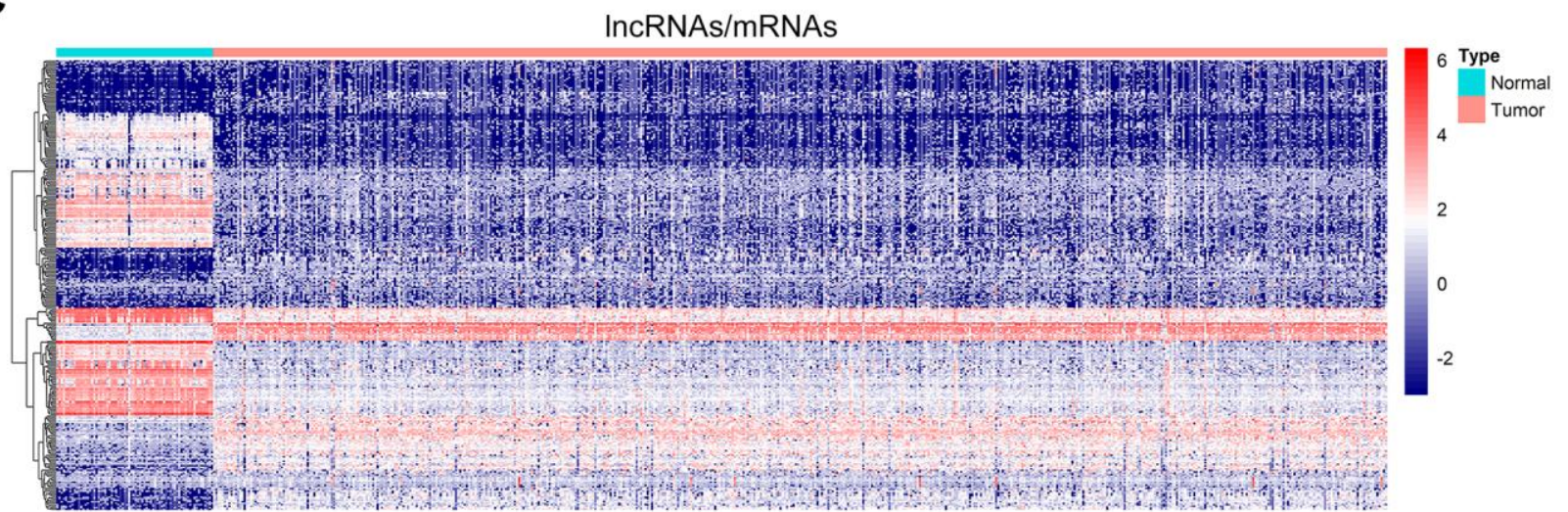

D

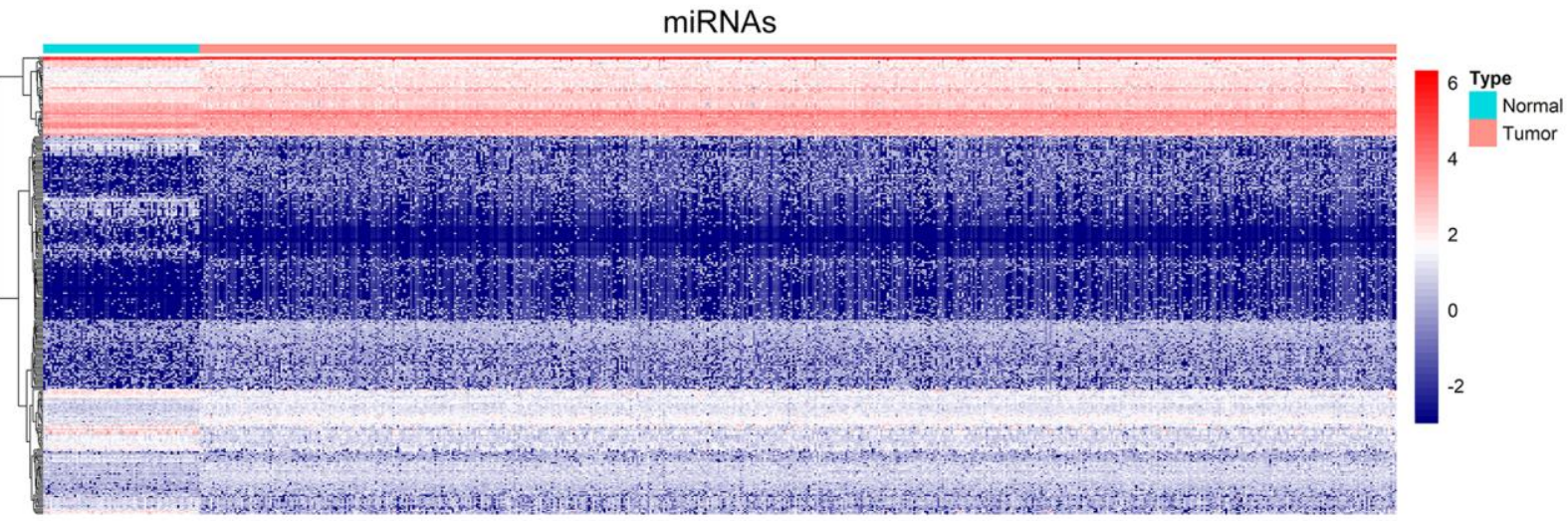

Figure 1. Differential gene expression analysis for ccRCC. (A, B) Volcano plot of the differentially expressed IncRNAs/mRNAs, and miRNAs. (C, D) The heatmap of the top 300 differentially expressed IncRNAs/mRNAs, and miRNAs. 
lncRNAs and mRNAs. The $\beta$ values were optimized, and $\beta=4\left(\mathrm{R}^{2}=0.86\right)$ was chosen as the optimum (Figure 3A, 3B). A total of 611 miRNAs were clustered into 13 modules (Figure 3C). Among these identified modules, the turquoise module, containing 83 miRNAs, was substantially related to ccRCC samples (Figure 3D). Additionally, a high GS-MM correlation (0.93) was identified in the turquoise module (Figure 3E).

\section{Construction of the ceRNA network}

We constructed the ceRNA network to investigate the regulatory mechanisms underlying lncRNAs in ccRCC. First, a total of 279 overlapping lncRNAs, 47 overlapping miRNAs, and 1040 overlapping mRNAs were identified through the intersection of differential gene expression analysis and WGCNA (Figure 4A, 4B). Then, the interactions between 1040 lncRNAs and 279 miRNAs were investigated using LncBase v.2 database. As a result, 457 interacting pairs of lncRNAs and miRNAs were identified, consisting of 173 lncRNAs and 45 miRNAs. Next, the three databases of TargetScan, miRDB, and miRTarBase were applied to assess mRNAs targeted by the 279 identified miRNAs in the previous stage. 73 interacting pairs of miRNAs and mRNAs were obtained, consisting of 23 miRNAs and 62 mRNAs. Finally, the above findings were applied to construct a lncRNAs-based ceRNA network. The ceRNA network is comprised of 144 lncRNAs, 23 miRNAs, and 62 mRNAs (Figure 4C).
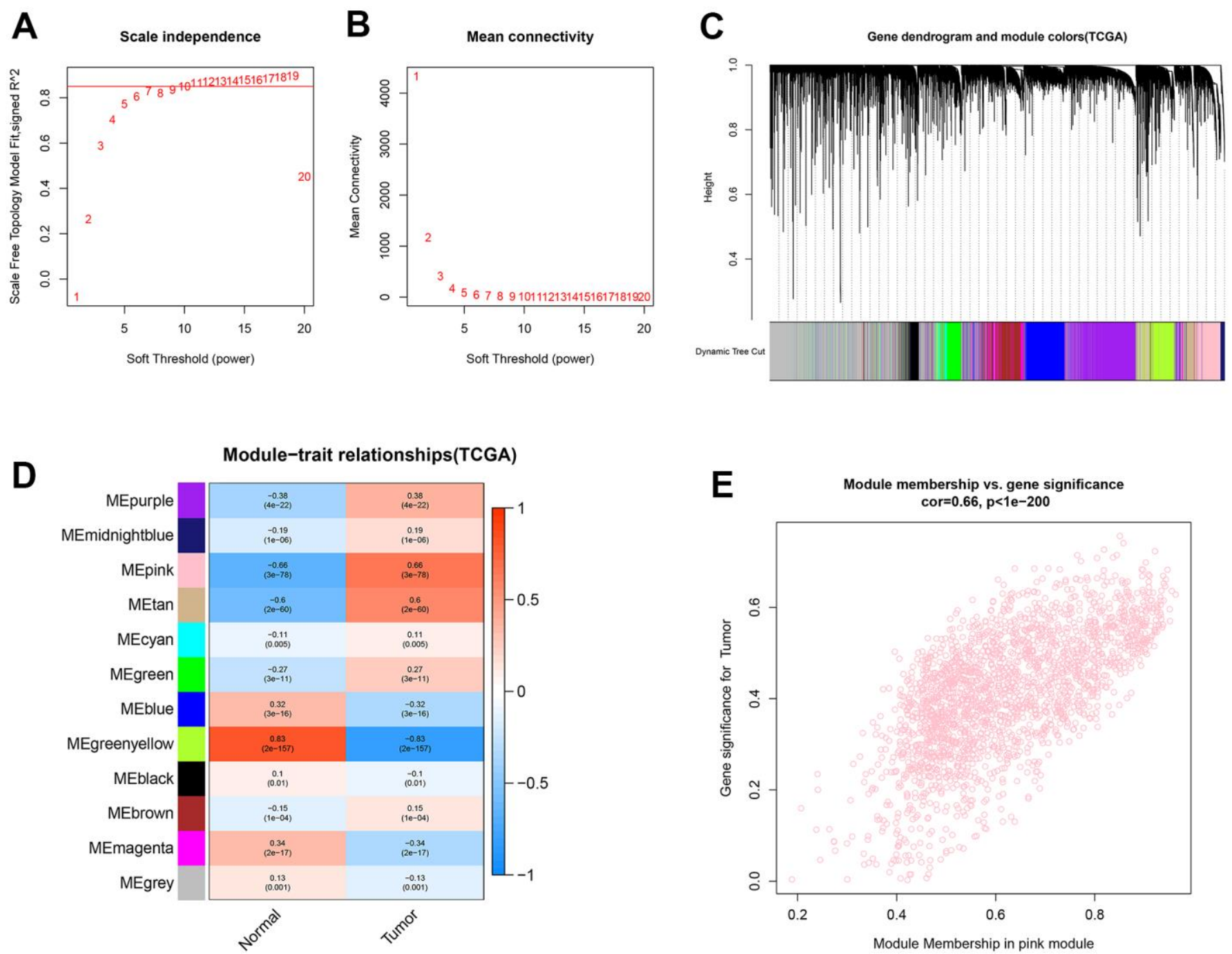

Figure 2. Identification of co-expression modules for IncRNAs/mRNAs based on WGCNA. (A, B) The scale-free fit index and the mean connectivity for various soft-thresholding powers ( $\beta$ ) were optimized. (C) Cluster dendrogram of IncRNAs/mRNAs based on the 1-TOM. (D) Heatmap of the correlation between module eigengenes and sample types. (E) Scatter plot of module eigengenes for ccRCC samples in the pink module. 


\section{Functional enrichment analysis for mRNAs in the ceRNA network}

GO and KEGG analyses were applied by utilizing STRING database for better comprehension of the potential biological functions and roles of the extracted mRNAs in the ceRNA network. In this regard, the terms "binding", "protein binding", "sequence-specific DNA binding", and "RNA polymerase II transcription regulatory region sequence-specific DNA binding" were identified as significant under molecular function (MF). In addition, the mRNAs were mainly enriched in "core-binding factor complex", "plasma membrane", "external side of plasma membrane", and "cell surface" in terms of cellular component (CC). Finally, the mRNAs related to biological process (BP) were most relevant in immune-related pathways, including "immune system process", "regulation of immune system process", "positive regulation of immune system process", and "immune response" terms (Figure 5A). According to the KEGG pathway analysis, mRNAs became significantly enriched in "pathways in cancer", "necroptosis", "transcriptional misregulation in cancer", "cytokine-cytokine receptor interaction", and "chemokine signaling pathway" (Figure 5B).

\section{Development of a prognostic risk model by lncRNAs in the ceRNA network}

At first, ccRCC samples were classified in random groups for the training $(\mathrm{n}=257)$ and the testing $(\mathrm{n}=256)$
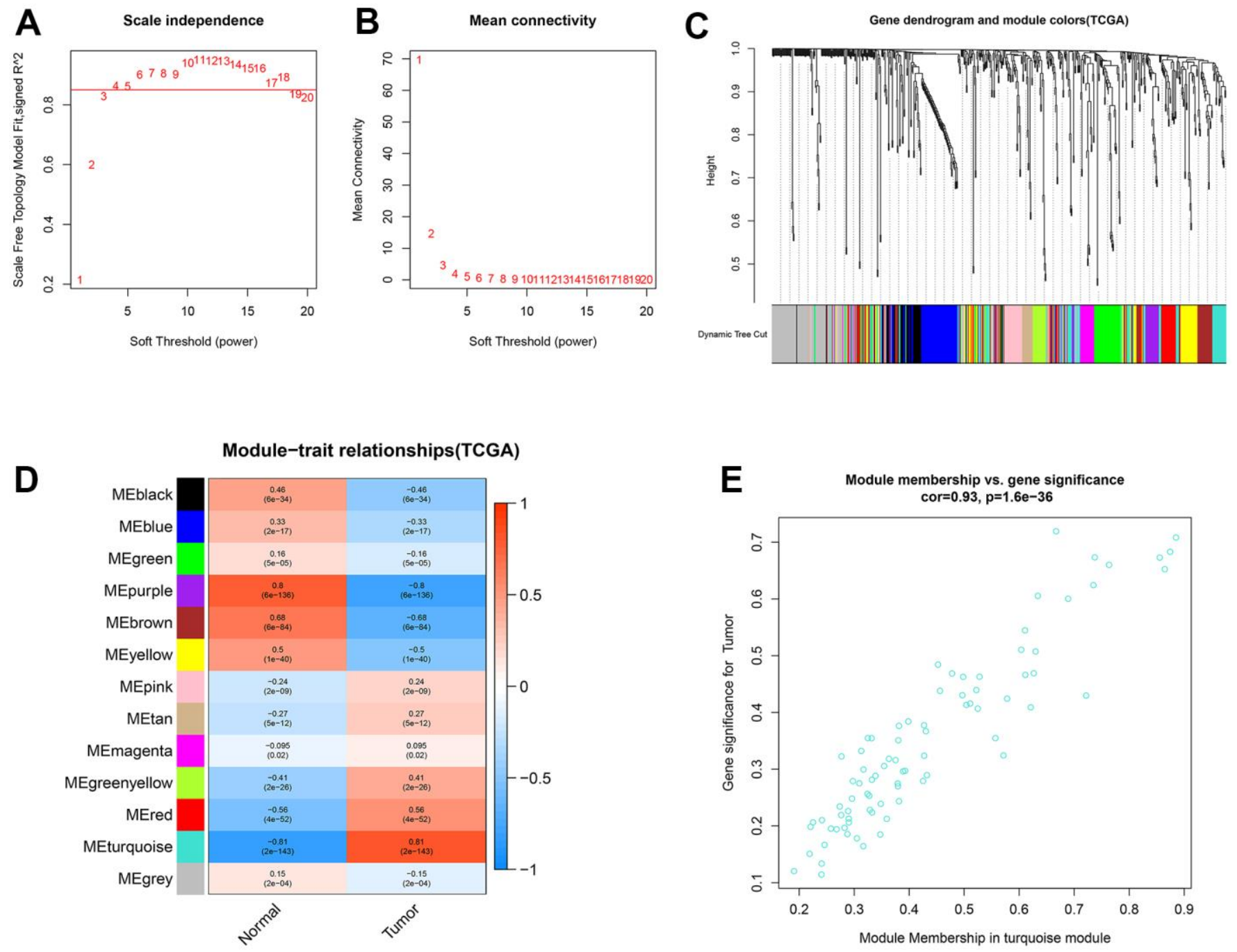

Figure 3. Identification of co-expression modules for miRNAs by WGCNA. (A, B) The scale-free fit index and the mean connectivity for various soft-thresholding powers ( $\beta$ ) were optimized. (C) Cluster dendrogram of miRNAs based on the 1-TOM. (D) Heatmap of the correlation between module eigengenes and sample types. (E) Scatter plot of module eigengenes for ccRCC samples in the turquoise module. 
A

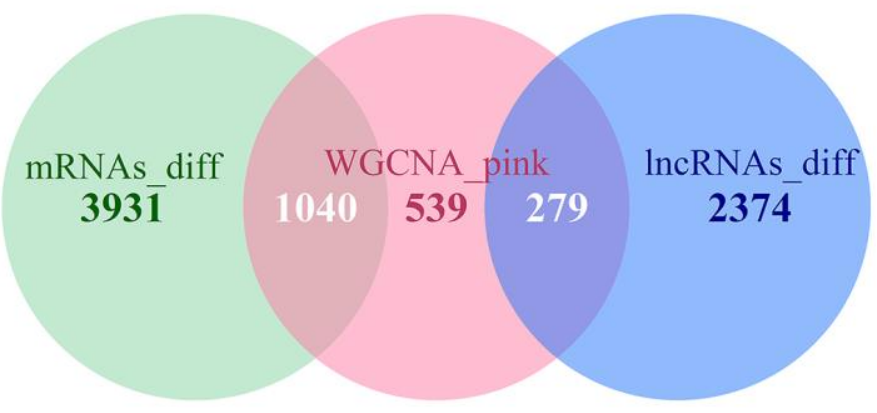

B miRNAs

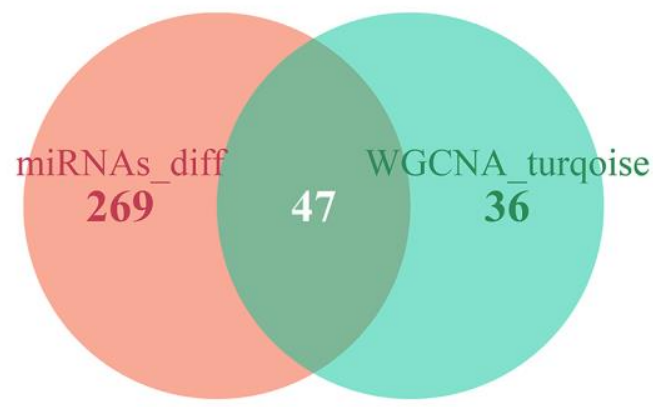

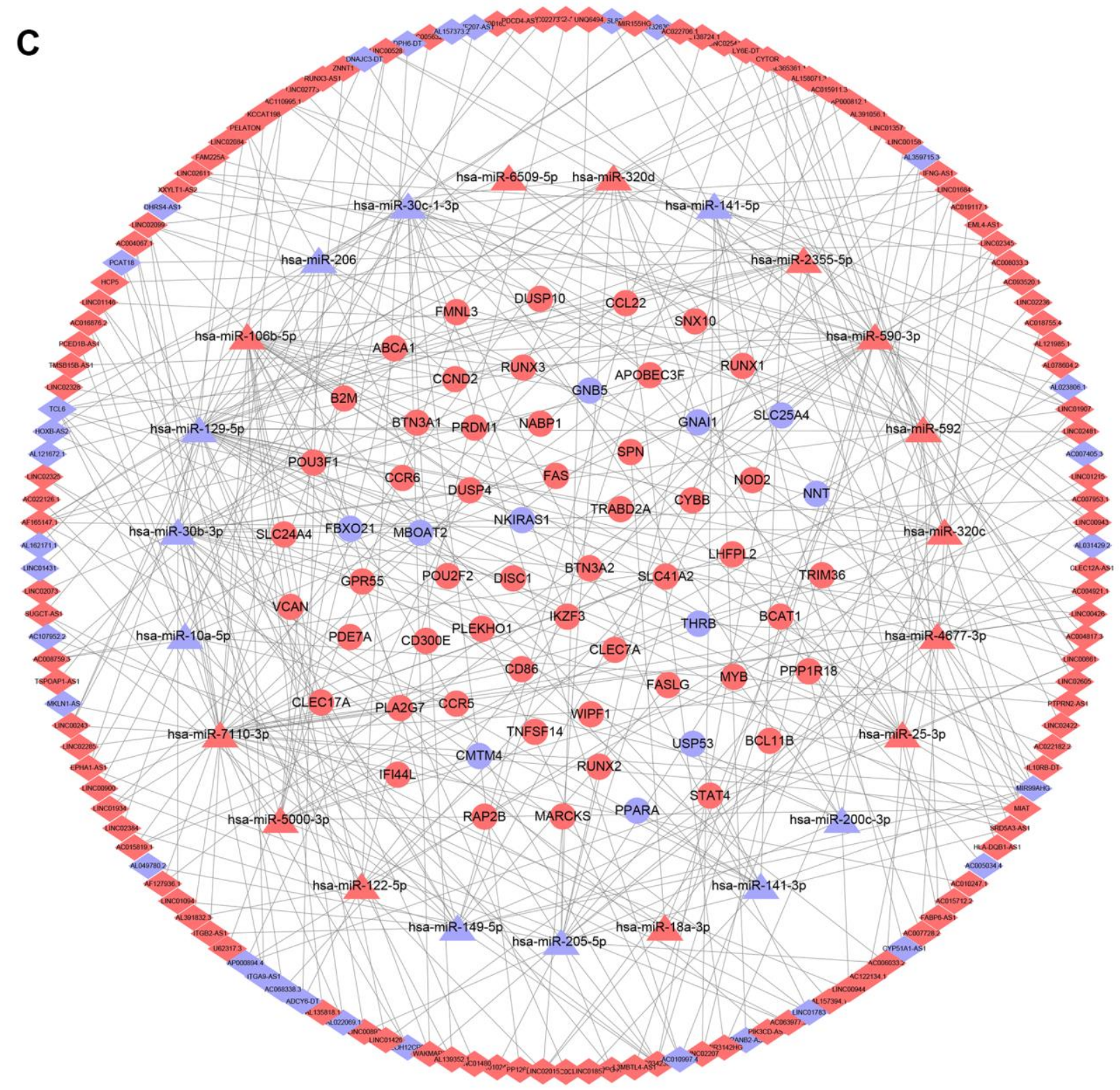

Figure 4. Construction of the ceRNA network in ccRCC. (A) Overlapping mRNAs and IncRNAs between differential gene expression analysis and WGCNA. (B) Overlapping miRNAs between differential gene expression analysis and WGCNA. (C) The ceRNA network was comprised of 144 IncRNAs, 23 miRNAs, and 62 mRNAs; red nodes represent up-regulation, and blue nodes represent down-regulation; diamond nodes represent IncRNAs, triangle nodes represent miRNAs, and ellipse nodes represent mRNAs. 
cohorts. Then, we developed the prognostic risk model using the training cohort. A total of 144 lncRNAs in the ceRNA network were evaluated via the univariate Cox regression analysis. With the threshold of $p<0.001,16$ lncRNAs were selected for further least absolute shrinkage and selection operator (LASSO) regression analysis. Eight lncRNAs were identified using lambda.min value (Figure 6A-6C), and then were integrated into the multivariate Cox regression analysis. Four lncRNAs with strong prognostic values were identified in total: LINC00943, SRD5A3-AS1, LINC02345, and U62317.3 (Figure 6D). Using these four lncRNAs, a prognostic risk model was developed with the calculated risk score $=(0.122 *$ LINC00943 expression level $)+(0.422 *$ SRD5A3-AS1 expression level $)+(0.282 *$ LINC02345 expression level $)+(0.247$ * U62317.3 expression level).

\section{Evaluation of the prognostic signature in the training cohort}

For this purpose, each ccRCC sample was evaluated in terms of the risk score. The training cohort samples were categorized into the high- $(\mathrm{n}=128)$ and low-risk $(\mathrm{n}=129)$ groups using the median risk score $=4.575$. Kaplan-Meier survival analysis revealed a significantly higher overall survival (OS) in the lowrisk group compared to the high-risk one (Figure 7A). The predictive capacity of this prognostic risk model and clinical parameters were evaluated using the receiver operator characteristic (ROC) curves. The area under the curves (AUCs) of risk scores for 1year, 5-year, and 10-year survival were 0.760, 0.736, and 0.842 , respectively (Figure 7B). Risk score, age, gender, grade, and stage AUCs at 5 years were 0.726,
0.509, 0.519, 0.669, 0.728, respectively (Figure 7C). The survival risk plot showed a poorer prognosis in the high-risk group. In other words, prognosis deteriorated with an increased risk score (Figure 7D, $7 \mathrm{E})$. Both the principal component analysis (PCA) and $\mathrm{t}$-distributed stochastic neighbor embedding ( $\mathrm{t}-\mathrm{SNE}$ ) test showed samples from different risk groups were distributed in different sections (Figure 7F, 7G).

\section{Evaluation of the prognostic signature in the testing cohort}

Using the median risk score of the training cohort, the ccRCC samples in the testing cohort could be categorized as the high- $(\mathrm{n}=142)$ and low-risk $(\mathrm{n}=$ 114) groups. Consistent with the previous results, the prognosis in low-risk samples was better than that of the high-risk samples (Figure 8A). The AUCs of risk scores for 1-year, 5-year, and 10-year survival were $0.701,0.742$, and 0.774 , respectively (Figure $8 \mathrm{~B}$ ). The predictive performance of the present risk score was more precise than clinical parameters and even other previous models [20-23] (Figure 8C, Supplementary Figure 1). The survival risk plot confirmed a more favorable prognosis in the low-risk group compared to the high-risk one (Figure 8D, 8E). The PCA and t-SNE analyses distributed the groups with distinct risk scores into two different categories (Figure 8F, 8G).

\section{Validation of independent prognostic factors}

To discover if the risk score acts as an independent prognostic clinical parameter, the univariate and multivariate Cox regression analyses were performed.
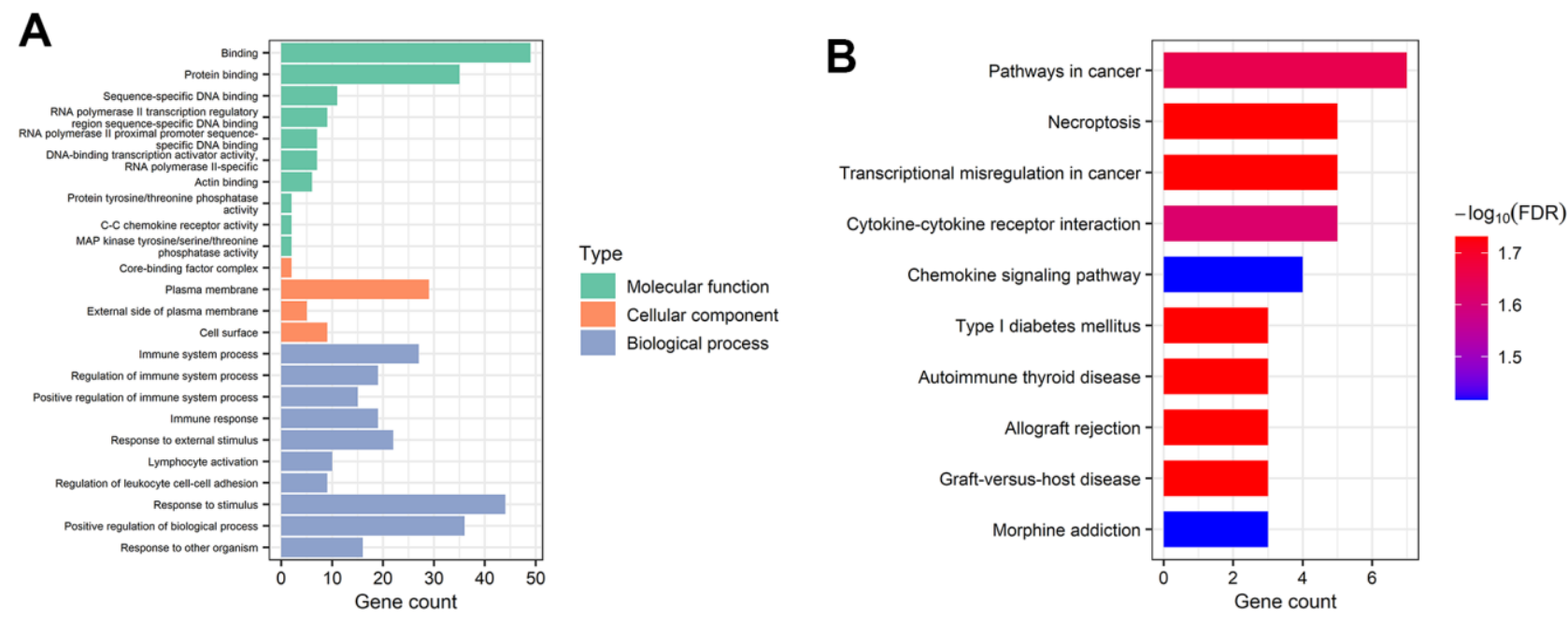

Figure 5. Gene functional enrichment analysis of mRNAs in the ceRNA network. (A) Overrepresented GO terms for MF, CC, and BP. (B) The top 10 functionally enriched pathways were identified by KEGG analysis. 
The results confirmed that the risk score and the stage as genuine independent prognostic OS predictors in both training cohort (Figure 9A, 9B) and testing cohort (Figure 9C, 9D).

\section{Identification of the genes related to the high-risk group}

WGCNA was applied to identify the genes related to the high-risk group. For this purpose, a soft-threshold $\beta=7\left(\mathrm{R}^{2}=0.86\right)$ was opted for developing a scale-free network (Figure 10A, 10B). Accordingly, 16 modules were identified to have a positive correlation with the high-risk group, among which the magenta module had the highest correlation value (Figure 10C, 10D). The correlation between MM and GS was 0.63 (Figure 10E). Finally, 1390 mRNAs in the magenta module were selected for functional enrichment analysis.

\section{Functional enrichment exploration of the high-risk group related genes}

To disclose the functions and pathways involved in samples of the high-risk group, GO and KEGG pathway enrichment analyses were applied on genes that were previously indicated to have the highest association with the high risk of ccRCC. BP terms included "immune system process", "immune response", and "regulation of immune system process" (Figure 11A). The genes related to CC were enriched in "plasma membrane", "cell periphery", and "side of membrane" (Figure 11B). In MF, the genes were involved in "immune receptor activity", "signaling receptor activity", and "transmembrane signaling receptor activity" (Figure 11C). Pathway analysis with KEGG illustrated that genes in "cytokine-cytokine receptor interaction", "chemokine signaling pathway", and
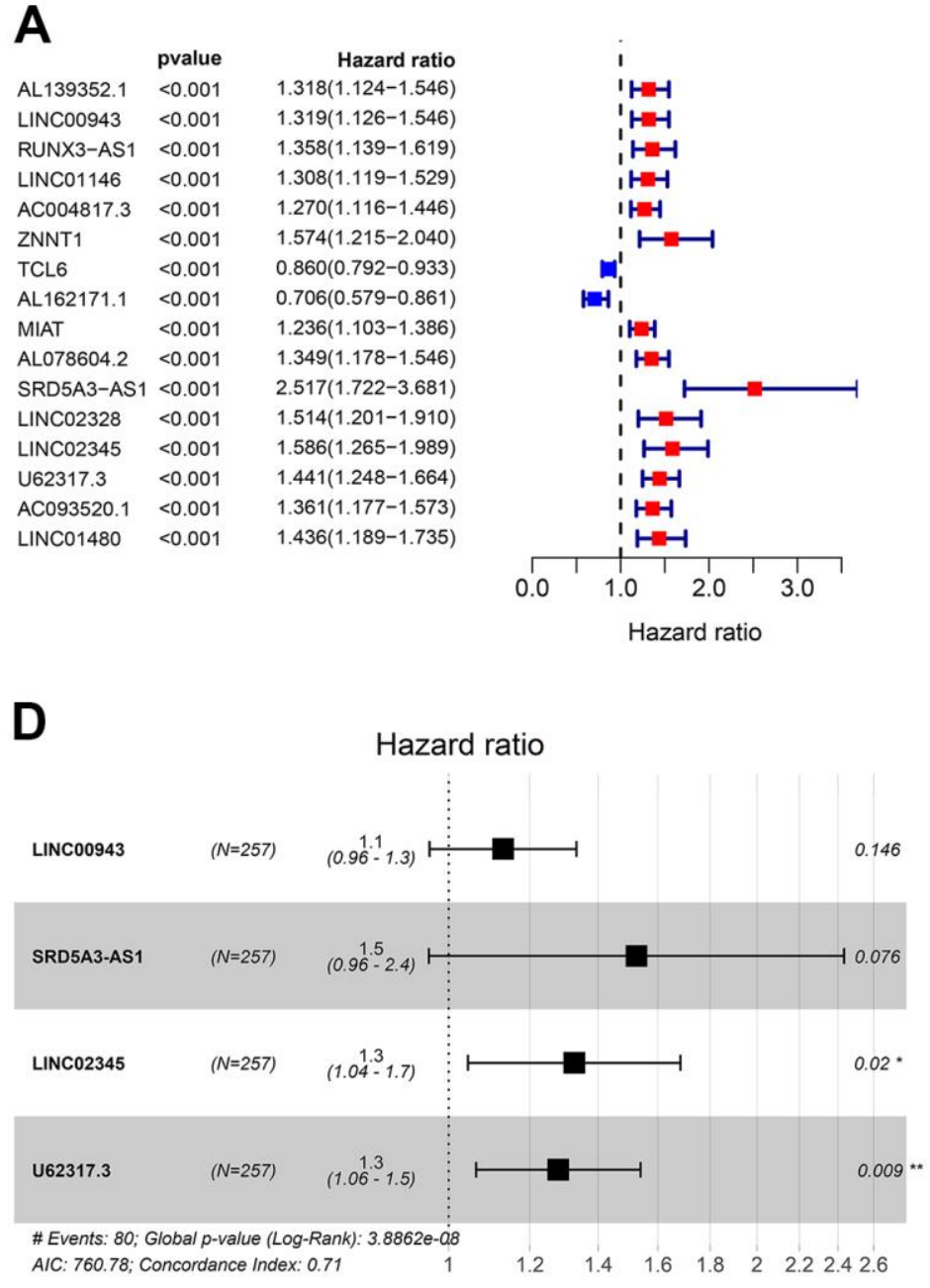

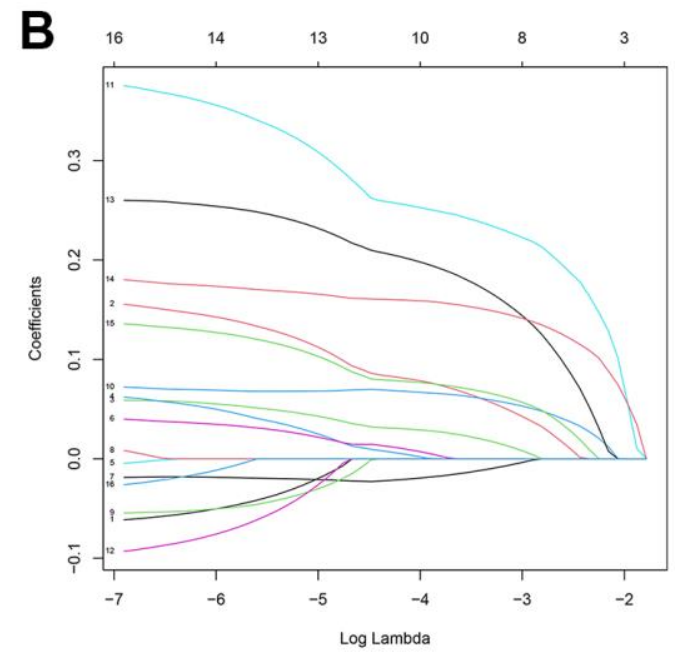

C

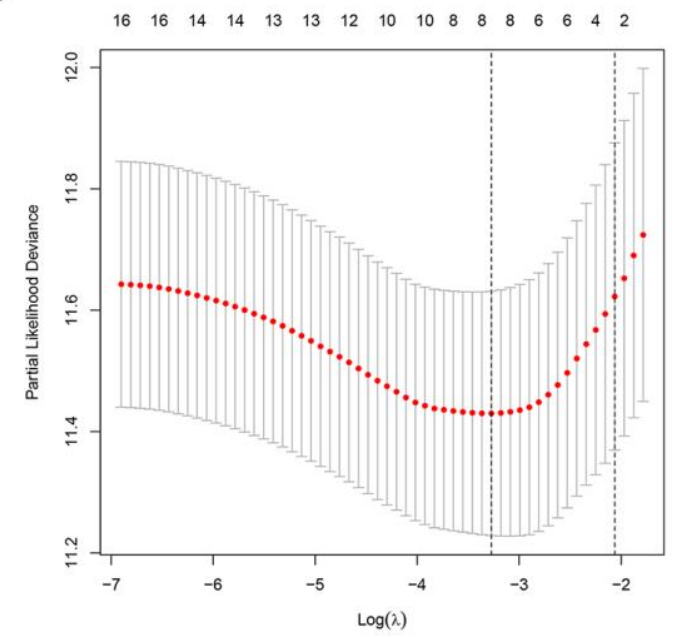

Figure 6. Construction of the prognostic risk model. (A) The results of the univariate Cox regression analysis of IncRNAs with $p<0.001$. (B, C) Lambda. min value $=8$ was calculated using LASSO regression. (D) The results of multivariable Cox regression analysis. 
"natural killer cell-mediated cytotoxicity" were significantly enriched (Figure 11D).

\section{Identification of immune cell infiltration in ccRCC for different risk scores}

The association between the infiltration levels of immune cells and the risk score was investigated by measuring the abundance of 22 different types of immune cells in ccRCC samples using the CIBERSORT algorithm (Figure 12A). The differential distribution of immune cell types between the groups with high and low risks of ccRCC was also examined. The results indicated a significantly greater proportion of infiltration of plasma cells, CD4 memory-activated $\mathrm{T}$ cells, and regulatory $\mathrm{T}$ cells
A

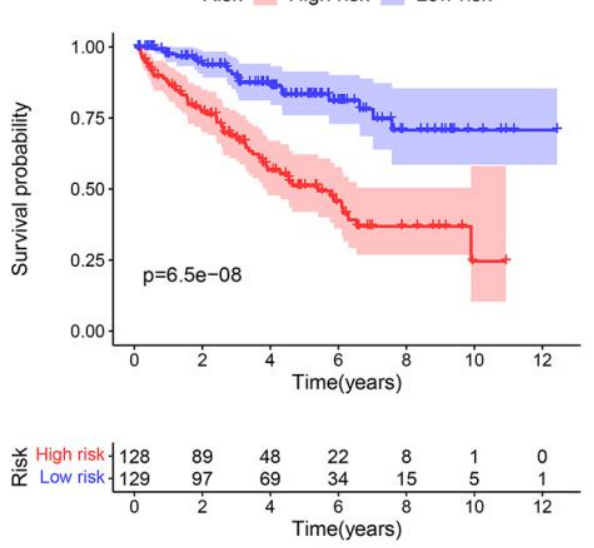

D

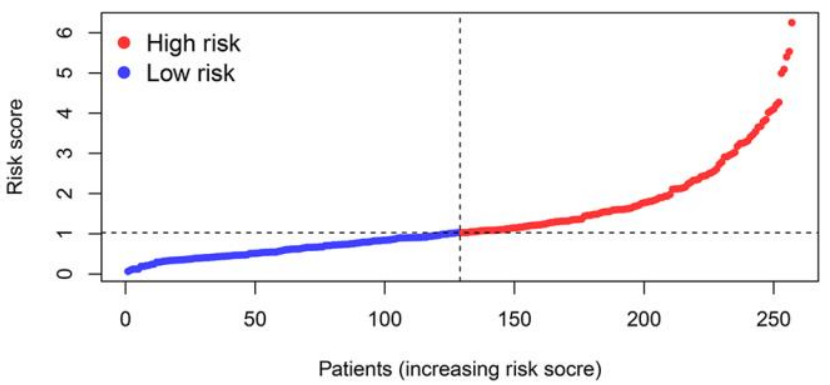

$\mathbf{F}$

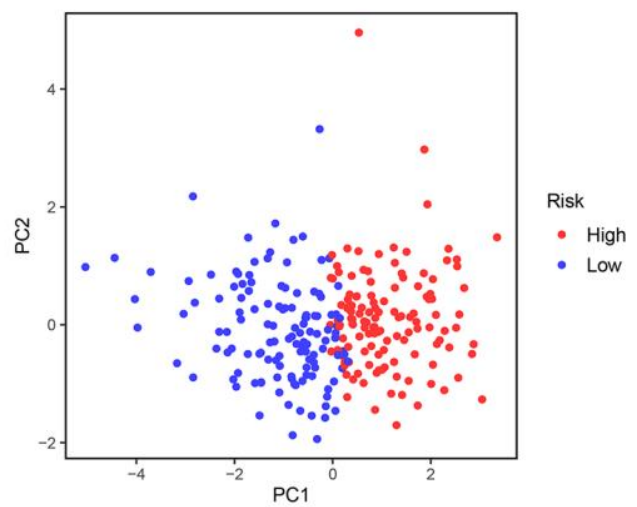

B

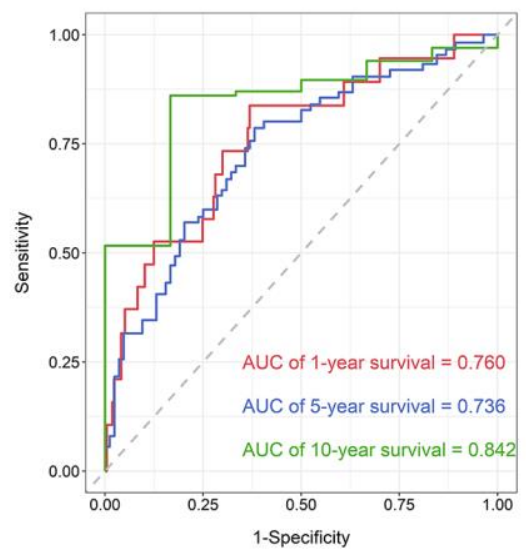

C

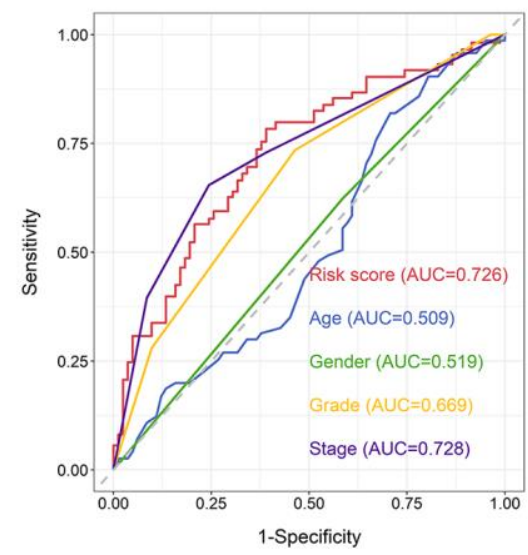

$\mathbf{E}$

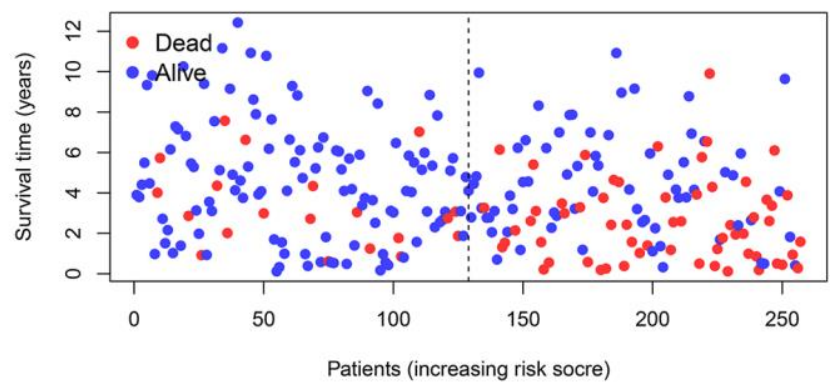

G

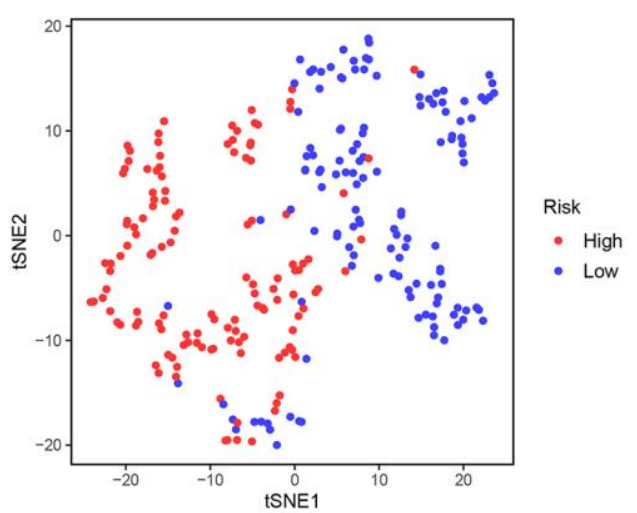

Figure 7. The predictive ability of the prognostic risk model in the training cohort. (A) The OS in the high- and low-risk groups. (B) ROC curves based on the prognostic risk model for predicting the 1-, 5-, and 10-year OS. (C) ROC curves based on the prognostic risk model and clinical parameters for predicting the 5-year OS. (D) Distribution of the risk score. (E) Correlation between the survival status and the risk score. (F) PCA for the high- and low-risk groups. (G) t-SNE analysis for the high- and low-risk groups. 


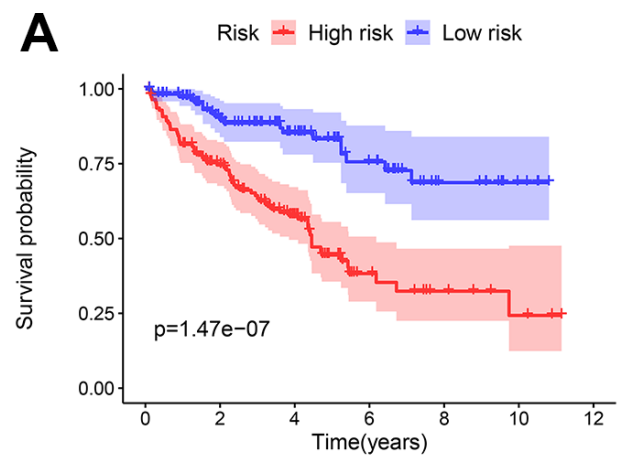

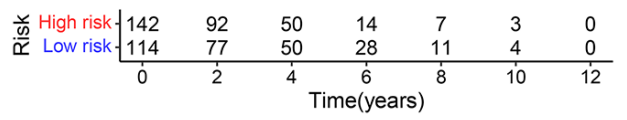
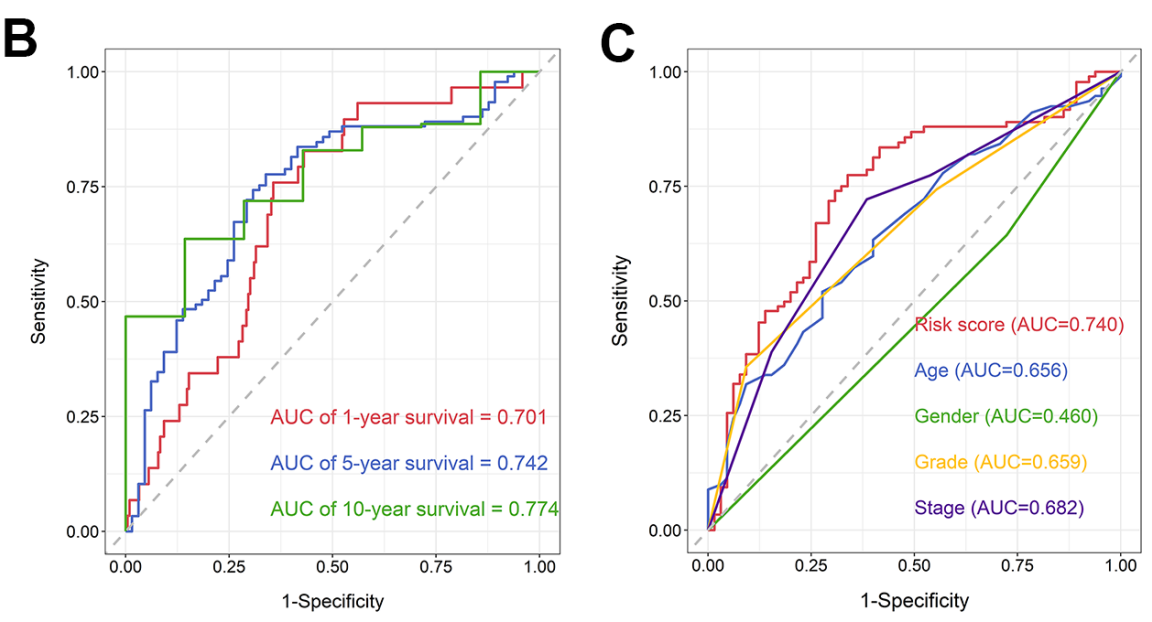

D

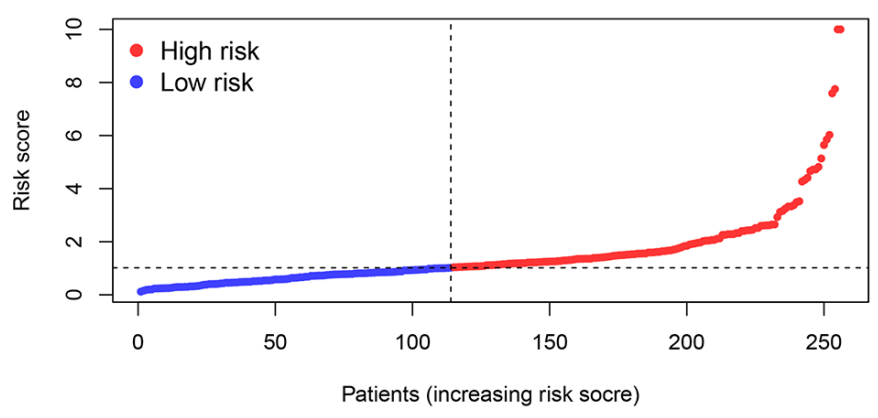

E

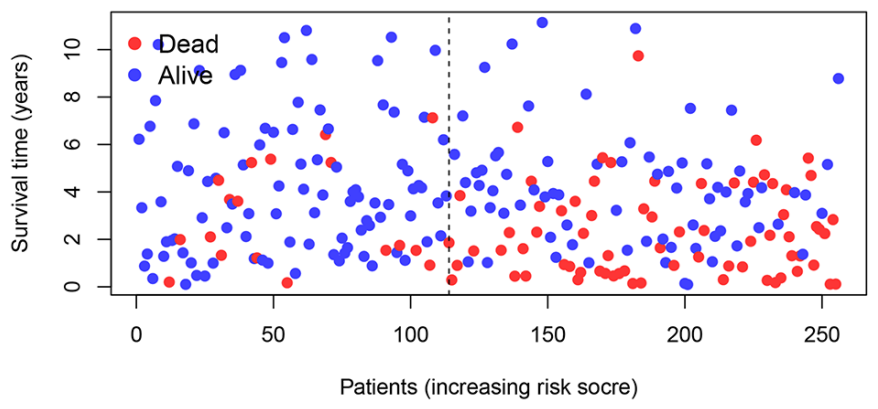

G

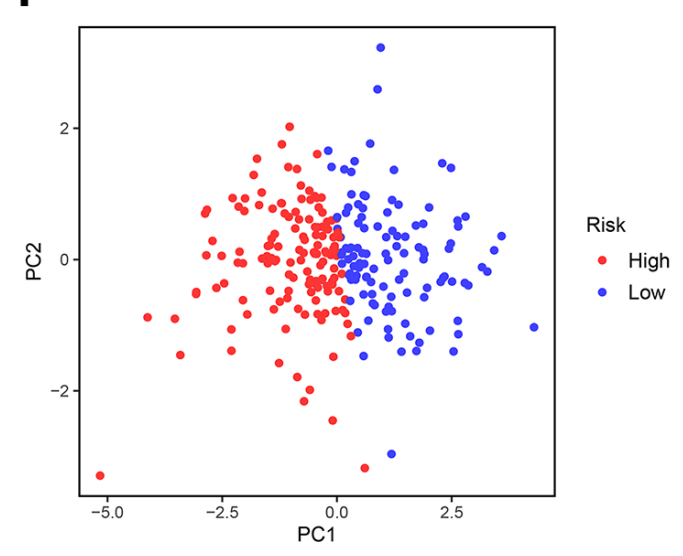

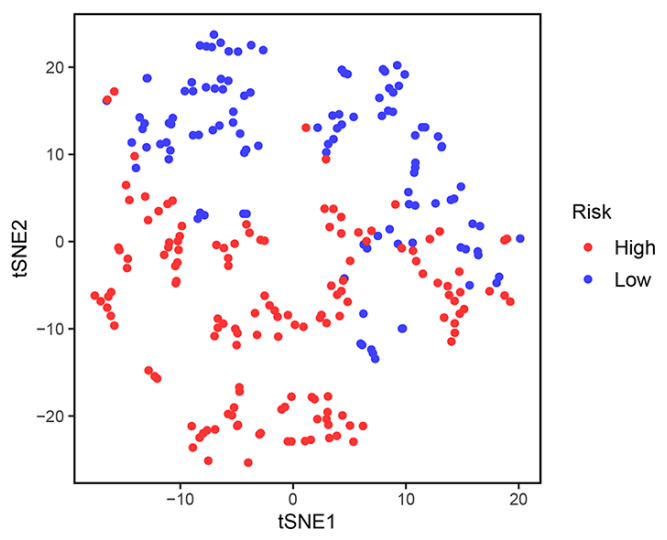

Figure 8. The predictive ability of the prognostic risk model in the testing cohort. (A) The OS in the high- and low-risk groups. (B) ROC curves based on the prognostic risk model for predicting the 1-, 5-, and 10-year OS. (C) ROC curves based on the prognostic risk model and clinical parameters for predicting the 5 -year OS. (D) Distribution of the risk score. (E) Correlation between the survival status and the risk score. (F) PCA for the high- and low-risk groups. (G) t-SNE analysis for the high- and low-risk groups. 
(Tregs) in the high-risk group compared to the low-risk group. In contrast, the infiltration of resting mast cells in the high-risk group was substantially lower than the same parameter in the low-risk group (Figure 12B).

\section{Identification of ICB-related gene expression for different risk scores}

Since ICB-related genes are widely known for their involvement in the development of cancers, the expressions of 47 ICB-related genes were also analyzed in the ccRCC samples with different risk scores. The expressions of multiple ICB-related genes in the low-risk group were significantly lower than that in the high-risk group (Figure 13A). Moreover, the expressions of most ICB-related genes and risk scores were positively correlated (Supplementary Table 1). The top six genes highly correlated with the risk score are shown in Figure 13B-13G.
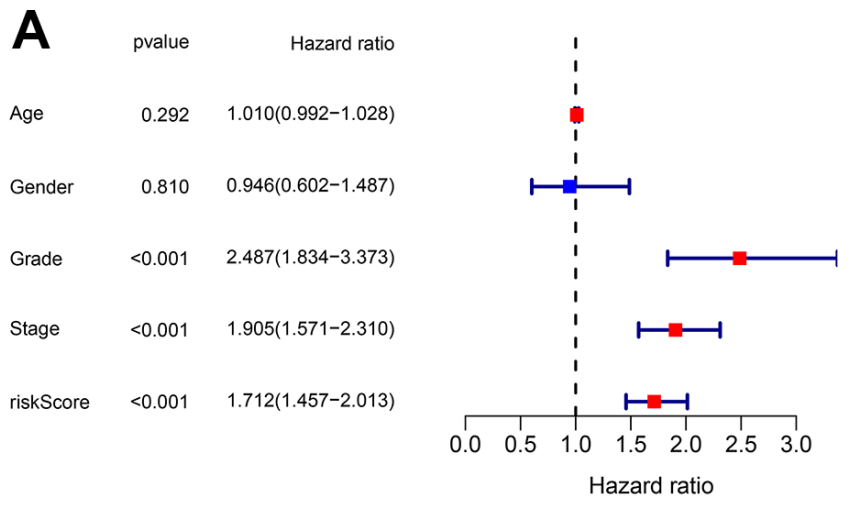

C
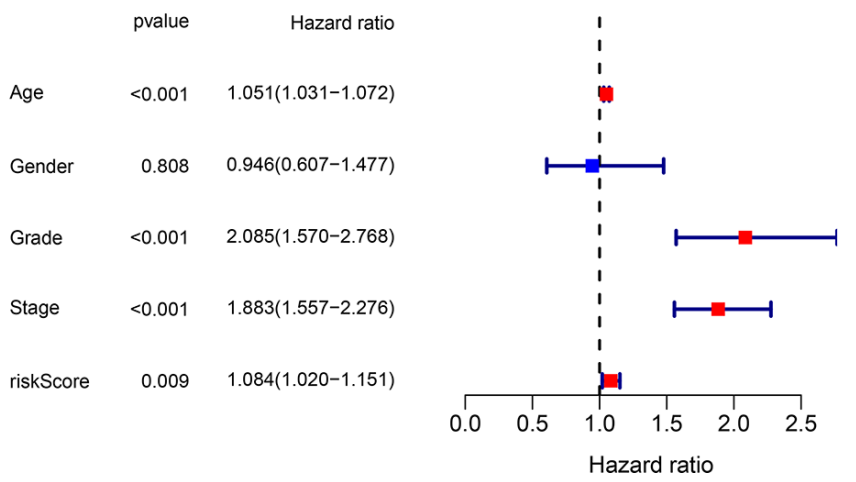

\section{DISCUSSION}

The recently developed high-throughput sequencing technologies have shifted the research focus from protein-coding RNAs to ncRNAs, especially lncRNAs. These molecules are being rapidly identified and characterized by the accumulating evidence to be strongly associated with tumorigenesis and progression in diverse cancers [24]. In addition, as compared to protein-coding RNAs, the relationship between lncRNAs and cancer status is tighter, which implies that IncRNAs may serve as accurate diagnostic, therapeutic, and prognostic biomarkers of several malignancies [25]. For instance, lncRNA SARCC expression in ccRCC tissues decreases, and low SARCC expression is associated with a worse prognosis. Androgen receptor has been identified as a target gene of SARCC. Knocking down SARCC decreases the expression of miR-143-3p and stimulates multiple oncogenes by enhancing androgen receptor expression, which leads to greater cancer cell proliferation, migration, and invasion

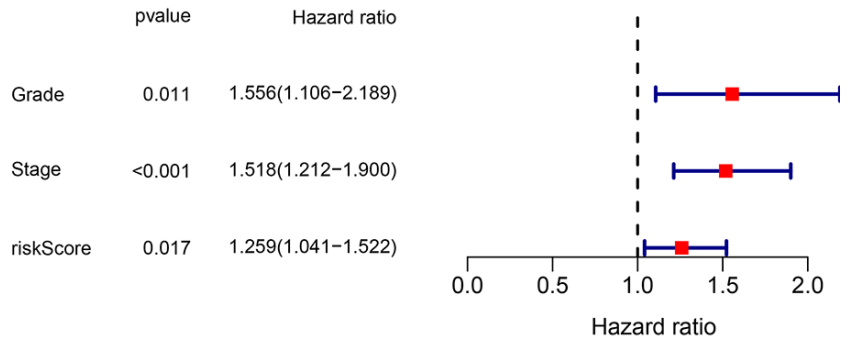

D

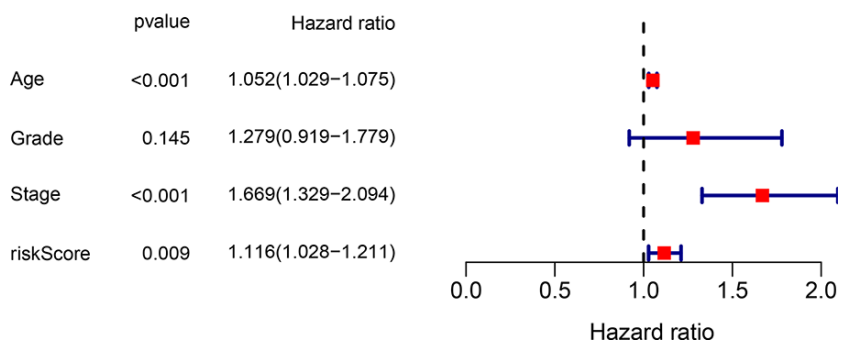

Figure 9. Validation of the independent prognostic factors. (A, B) Univariate and multivariate Cox regression analyses of the risk score and clinical parameters in the training cohort. (C, D) Univariate and multivariate Cox regression analyses of the risk score and clinical parameters in the testing cohort. 
[26]. Similarly, lncRNA MRCCAT1 is overexpressed in ccRCC cell lines as well as the relevant tissues. MRCCAT1 enhances the binding of PRC2 at NPR3 promoter regions, leading to transcriptional repression of NPR3 and the activation of the p38-MAPK signaling pathway. Thus, the aggressive cancerous cell phenotypes are enhanced both in vitro and in vivo [27]. Although previous studies have expanded our knowledge on lncRNAs to a certain extent, the potential functions and molecular mechanisms underlying lncRNA actions remain unknown.

WGCNA package in $\mathrm{R}$ is widely used for identifying the co-expressed gene modules based on the correlation within gene expression profiles as well as the correlation between gene modules and clinical characteristics. Thereby, the genes with analogous expression tendencies and functions are clustered into the separate gene modules, and those gene modules which are most relevant to clinical traits are representative of the prominent regulators of the disease $[28,29]$. The differential gene expression analysis and WGCNA are performed for genes associated with ccRCC, and the overlapping genes are considered to be pivotally involved in the development of ccRCC. To the best of our knowledge, the present report is the first study that employs the results of WGCNA to construct the ceRNA network in ccRCC. Thus, our results are reliable and convincing.

The role of immune system involvement in carcinogenesis is widely recognized. In immunocompetent individuals, the immune system takes responsibility for recognizing and exterminating the cancer cells [30]. However, emerging evidence suggests that evasion of immune
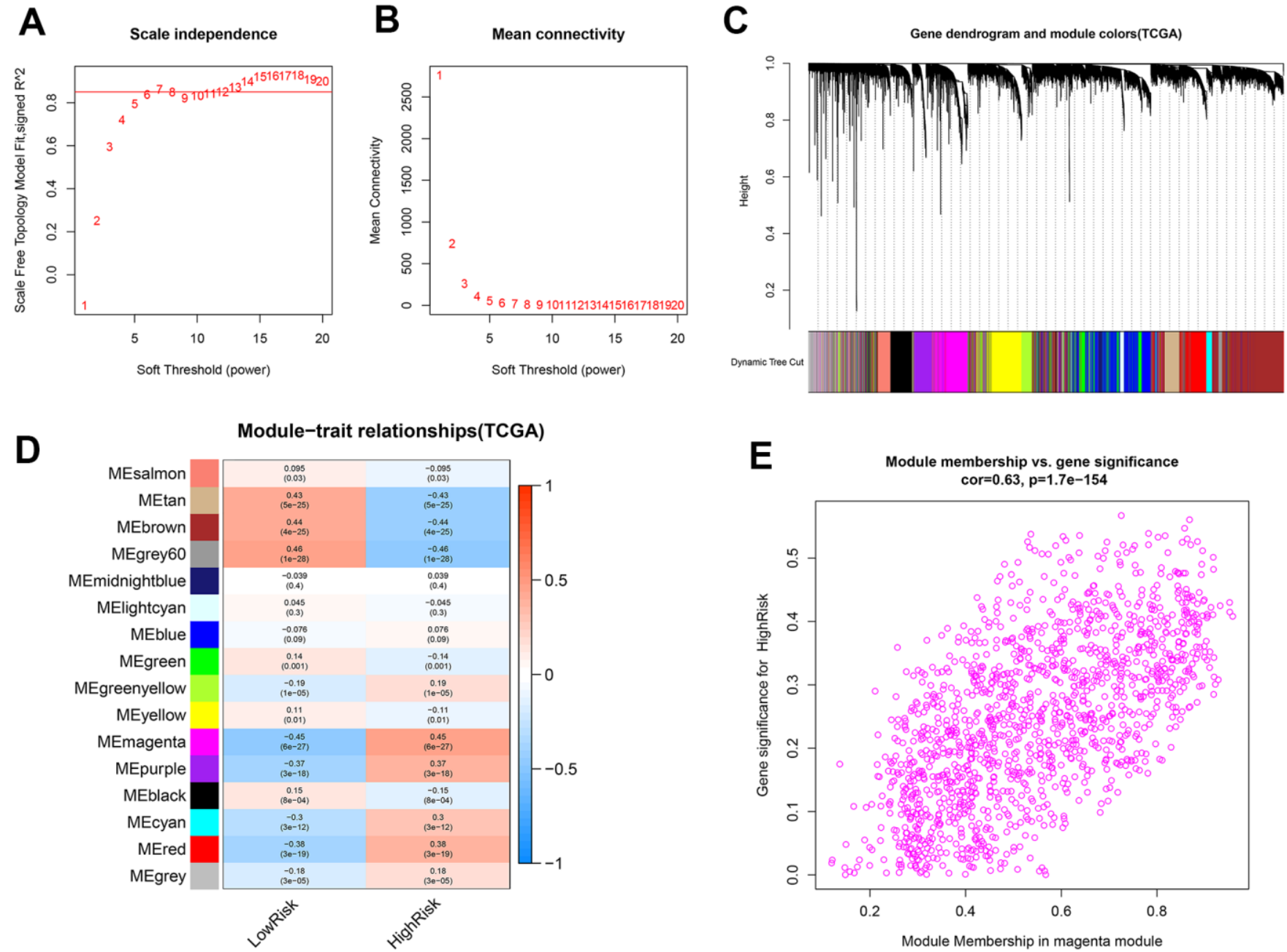

Figure 10. Identification of co-expression modules related to the high-risk group by WGCNA. (A, B) The scale-free fit index and the mean connectivity for various soft-thresholding powers $(\beta)$ were optimized. (C) Cluster dendrogram of genes based on the 1-TOM. (D) Heatmap of the correlation between module eigengenes and sample types. (E) Scatter plot of module eigengenes for the high-risk group in the magenta module. 
destruction is a distinct feature of cancers [31]. Hence, immunotherapy as a promising therapeutic strategy has attracted extensive research attention. Several immunotherapeutic biomarkers have been identified, and the corresponding immunotherapeutic agents have received approval to be used in clinical trials or treatment, including cytotoxic T-lymphocyte associated protein 4 (CTLA4) inhibitor, programmed cell death protein 1 (PD-1)/ PD-1 ligand 1 (PD-L1) inhibitor, and chimeric antigen receptor (CAR) T cell. Nevertheless, such treatments are currently accessible only for a specific subset of patients. Thus, the identification of novel immunotherapeutic biomarkers is necessary [32]. Accumulating studies suggest that lncRNAs may function as effective immunotherapeutic targets. Huang et al. [33] reported that activation-induced cell death of T-lymphocytes assists cancer cell evasion from immune
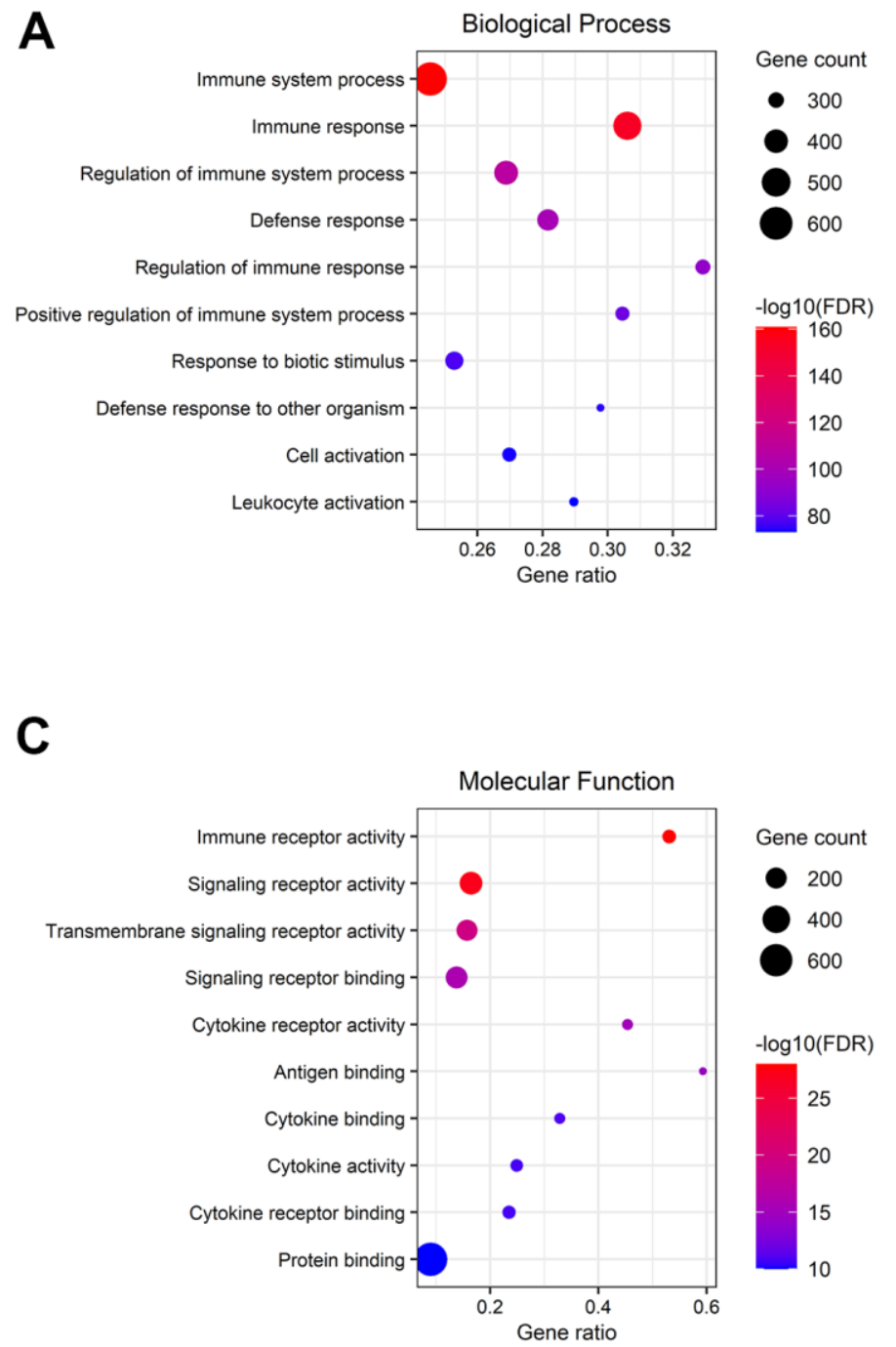

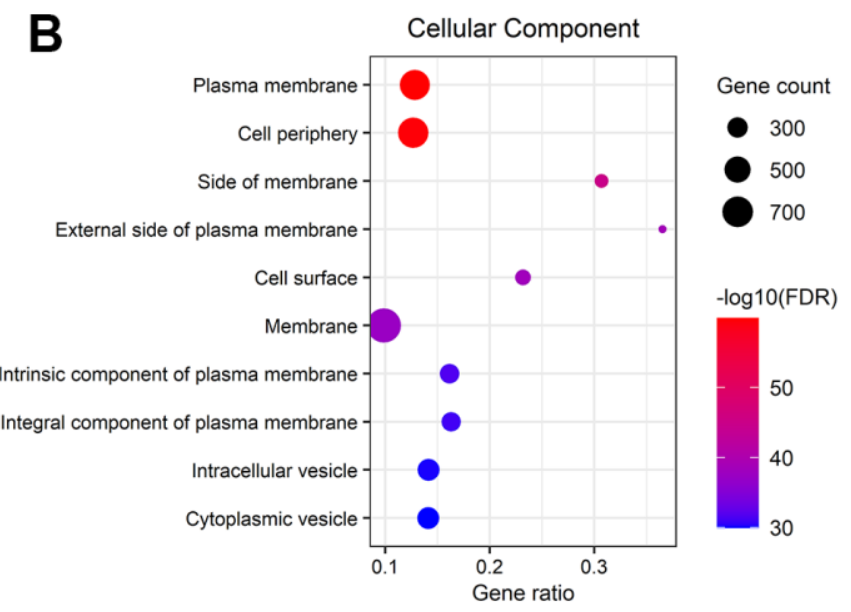

destruction. IncRNA NKILA overexpression sensitizes tumor-specific cytotoxic T-lymphocytes and type 1 helper $\mathrm{T}$ cells, resulting in activation-induced cell death and cancer immune evasion in breast and lung cancers. Liu et al. [34] reported that LINC00973 induces immune suppression by regulating the ceRNA network. Siglec-15, a novel immune suppressor, is promoted by LINC00973/ miR-7109 axis and leads to immune evasion in ccRCC. Consequently, these results imply that lncRNAs in the ceRNA network may regulate immune-related genes and provide novel immunotherapeutic targets. In our study, we identified four promising lncRNAs including LINC00943, SRD5A3-AS1, LINC02345, and U62317.3. A recent study shows that LINC00943 is upregulated in gastric cancer. Knocking down LINC00943 enhances the expression of miR-101-3p, resulting in the suppression of cell proliferation and chemoresistance.

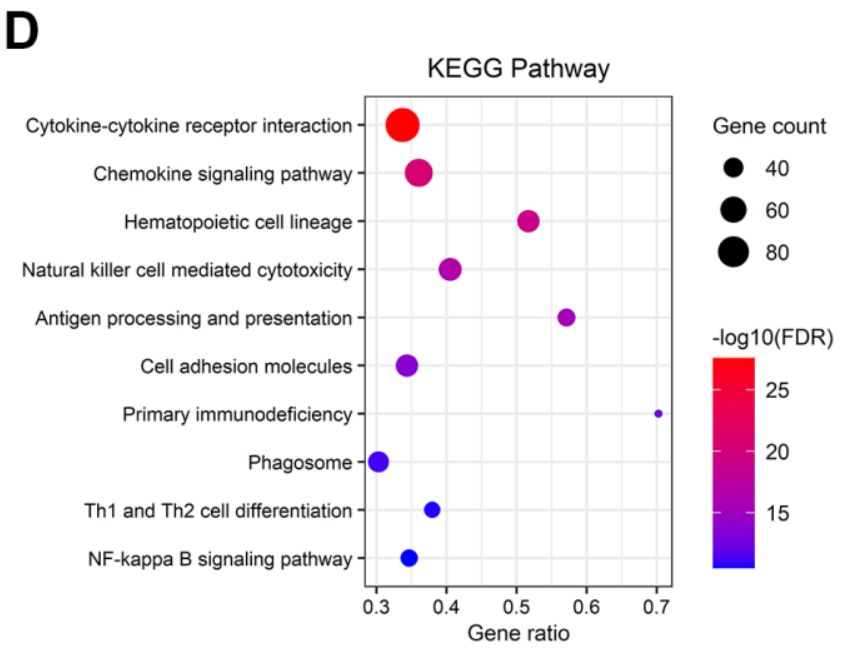

Figure 11. Gene functional enrichment analysis of genes associated with the high-risk group. (A-C) Overrepresented GO terms for BP, CC, and MF. (D) Top 10 enriched functional pathway terms based on KEGG analysis. 
However, SRD5A3-AS1, U62317.3, and LINC02345 have not yet been reported in cancers.

Immune cell infiltration is a pivotal component of TME, and dysregulation of immune cells has a powerful impact on clinical outcomes. The present results demonstrated that the immune cell infiltration is distinguished and distinctive in ccRCC samples belonging to different risk groups. In samples with high risk scores, plasma cells, CD4 memory-activated T cells, and Tregs were upregulated, while resting mast cells were downregulated. Several studies report high immune cell infiltration in ccRCC, especially that of the T cells
$[35,36]$. Upregulation of CD4 memory-activated T cells and Tregs showed to be negatively correlated with the OS [37]. Tregs are potent immunosuppressive cells that limit antitumor immunity and promote angiogenesis. Extensive Tregs infiltration is reported in multiple tumor types and is linked with poor clinical outcomes [38]. B cells can differentiate into plasma cells and produce antibodies, and are an important component of TME [39]. However, B cells recruited from TME promote RCC metastasis by activating the IL- $1 \beta / \mathrm{HIF}-2 \alpha /$ Notch 1 signaling axis [40]. The role of mast cells in ccRCC remains controversial. Some studies show that mast cell infiltration correlates with poor survival, since it

A

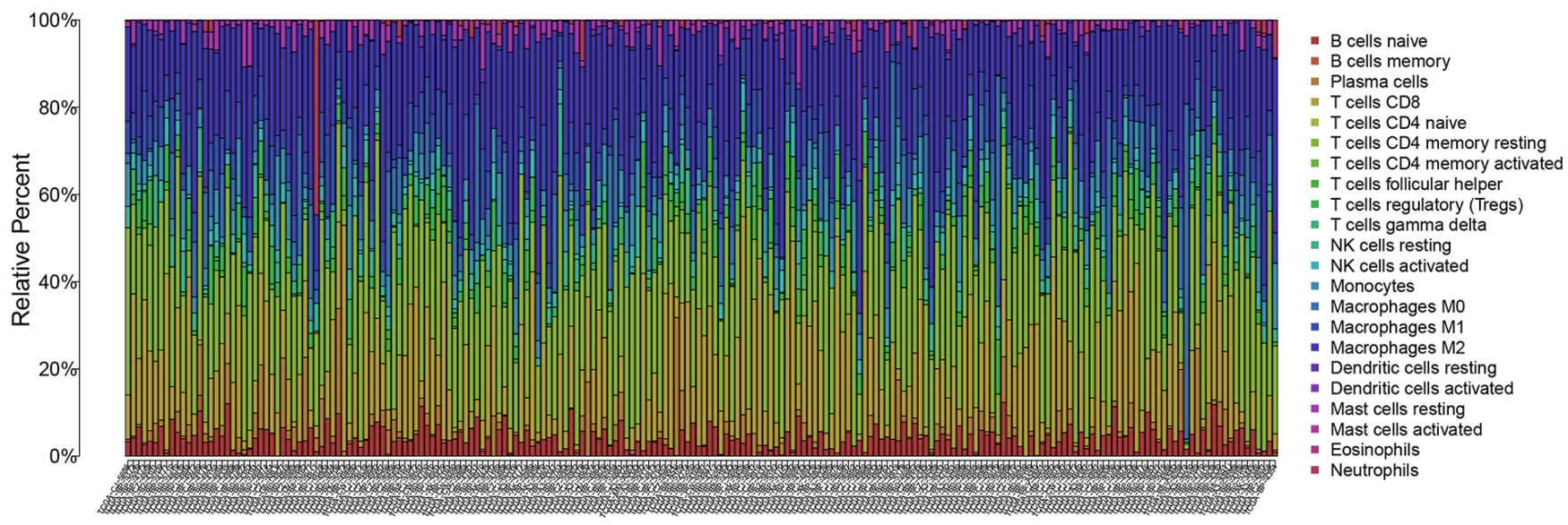

B

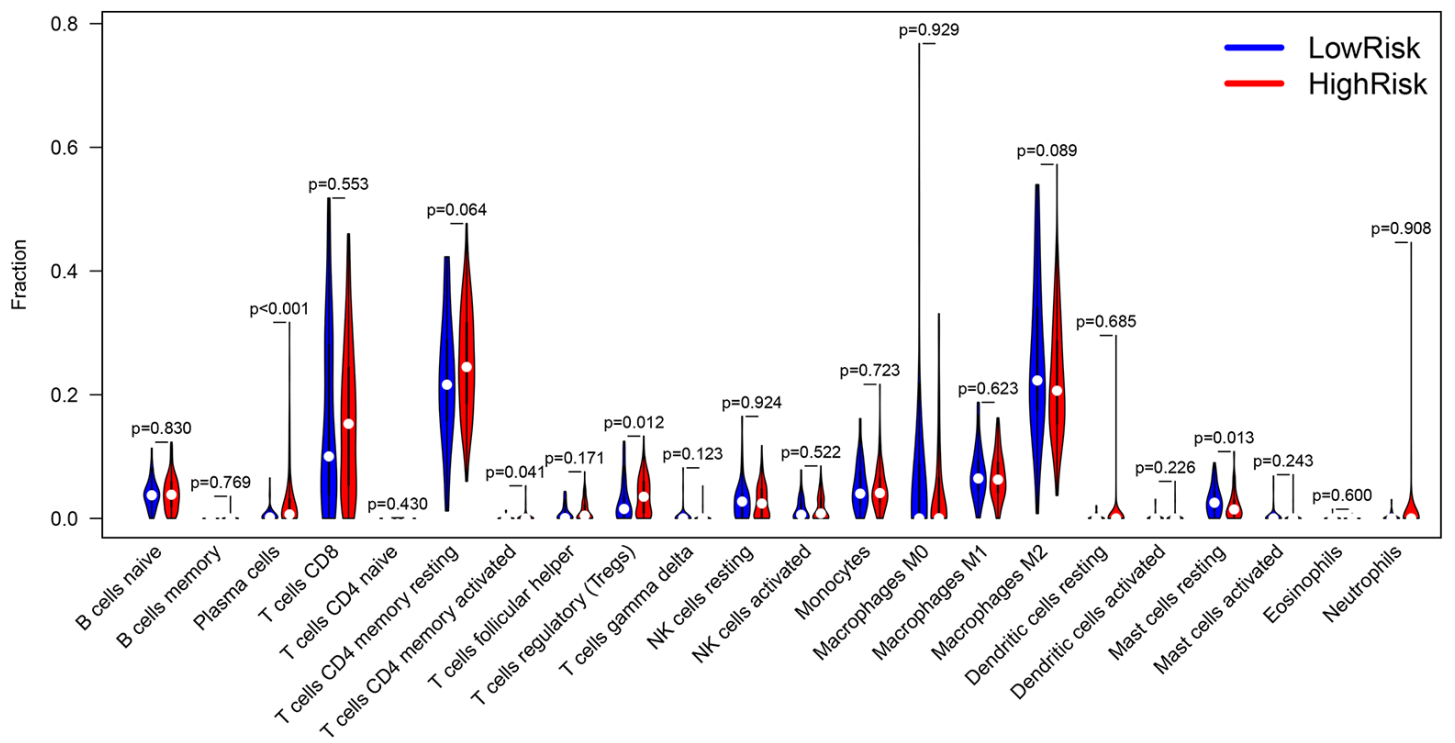

Figure 12. Evaluation of the immune cell infiltration in ccRCC samples. (A) The abundance of 22 immune cell types in ccRCC samples. (B) The proportion of different immune cell types between the high- and low-risk groups in ccRCC samples. 
has shown a positive correlation with the size, grade, and metastasis parameters of the tumor [41, 42]. Nevertheless, Xiong et al. [43] suggest that increased infiltration of mast cells represents high sensitivity to tyrosine kinase inhibitors treatment response and good survival. The controversy is reflective of the inherent complexity of TME in ccRCC, and more studies are required to elucidate and address the TME complexities in the future.

In conclusion, an immune signature-related ceRNA network was constructed, and a corresponding prognostic risk model was developed based on four lncRNAs with a significant prognostic value. To achieve this, the ccRCC samples have been categorized into the high- and low-risk groups according to the risk score. Further analyses indicated that the clinical outcome was significantly correlated with specific immune cell infiltration levels and ICBrelated gene expression in ccRCC samples belonging to different risk groups. Therefore, we envisage that our study offers a reference for identifying novel prognostic biomarkers and benefits studies aimed at developing immunotherapeutic strategies.
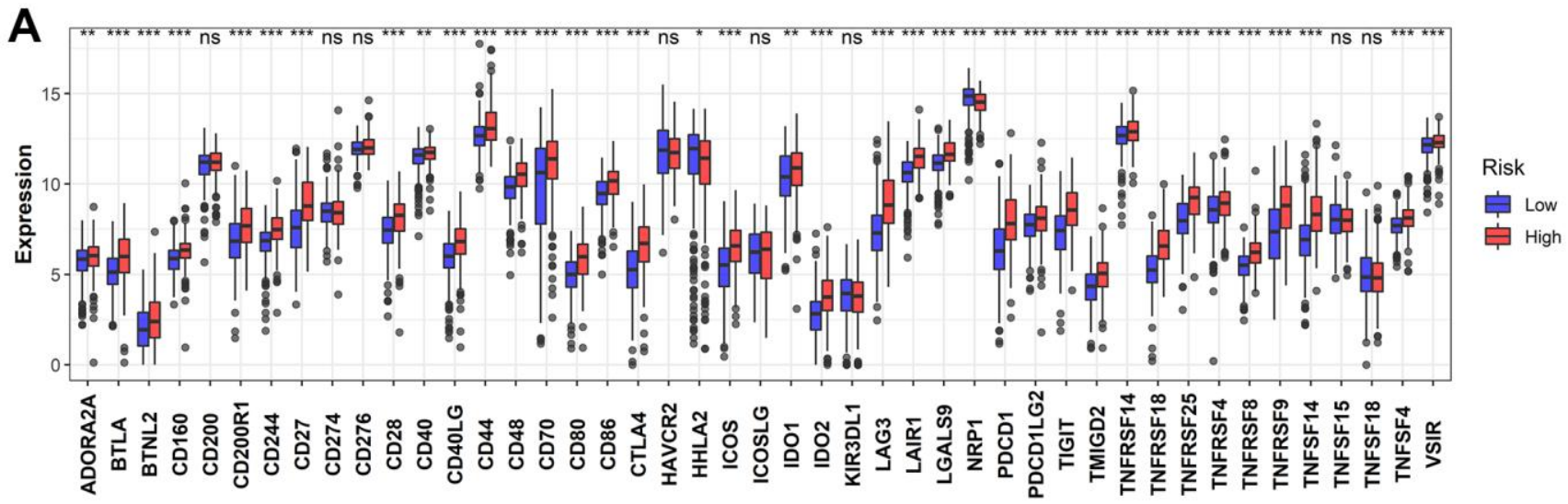

B

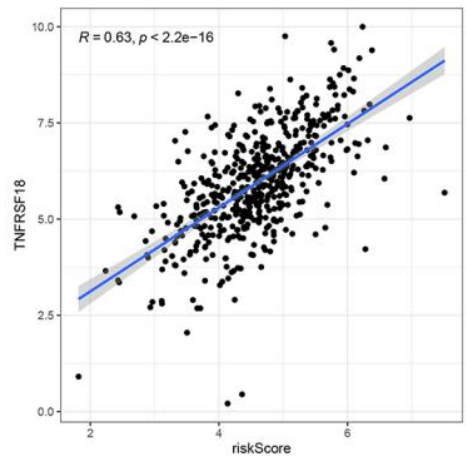

$\mathbf{E}$

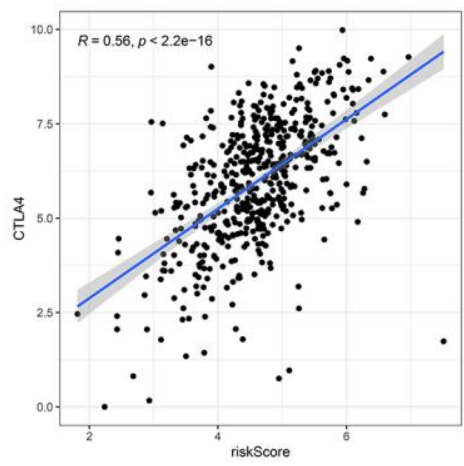

C

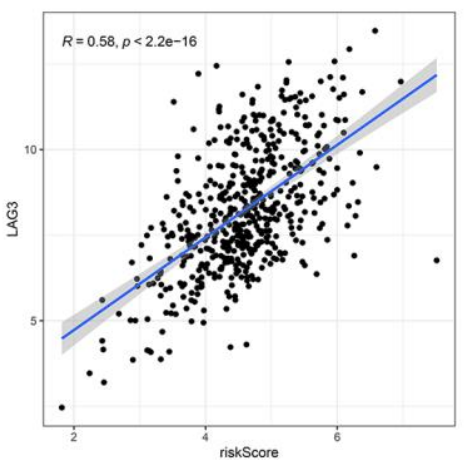

$\mathbf{F}$

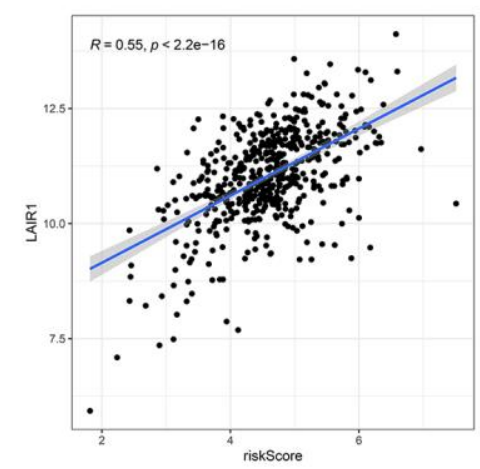

D

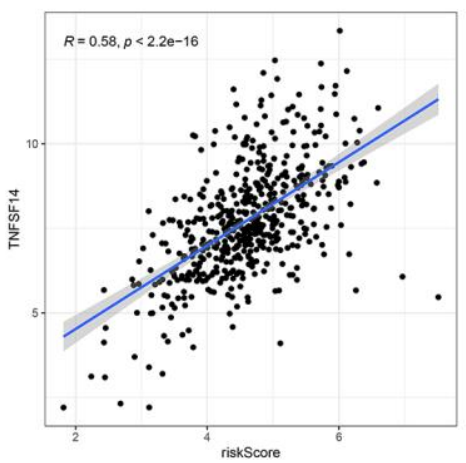

G

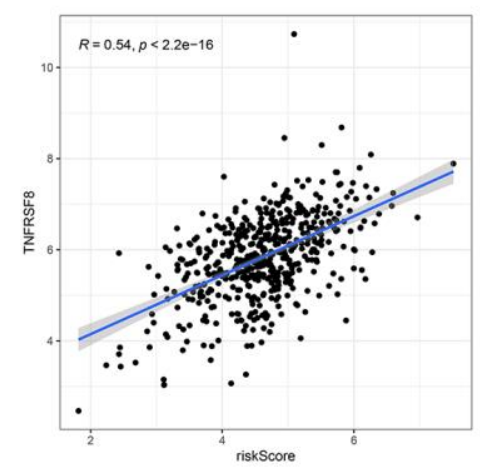

Figure 13. Identification of the correlation between the risk score and ICB-related genes. (A) The differential expression of ICBrelated genes between the high- and low-risk groups. (B-G) The top six ICB-related genes with the most relevance to the risk score. 


\section{MATERIALS AND METHODS}

\section{Data collection}

TCGA is a cancer genomics consortia-developed database comprising more than 20,000 primary cancer and correspondingly matched normal tissues from patients, spanning across 33 different cancer types. The TCGA portal (https://portal.gdc.cancer.gov/) was used to retrieve the RNA sequences and clinical data of ccRCC samples. The downloaded lncRNA and mRNA sequencing data comprised 539 ccRCC samples and 72 normal tissue samples of the kidney. The miRNA sequencing data comprised $545 \mathrm{ccRCC}$ and 71 normal tissue samples of the kidney. Since the required data were accessible from a public portal, no ethical approval or informed consent was needed.

\section{Differential gene expression analysis}

The gene sequencing data retrieved from TCGA were analyzed by the "edgeR" package [44] in the R software. The differentially expressed lncRNAs, miRNAs, and mRNAs were identified using $|\log 2 \mathrm{FC}|>1$ and FDR < 0.05 as the set threshold.

\section{Construction of weighted gene co-expression network}

WGCNA [45] was utilized to develop the gene coexpression modules gleaned from the clinical features. First, the gene expression profiles were normalized using the "edgeR" package. Then, data reliability confirmation was performed by checking the sample quality using the goodSamplesGenes function in the WGCNA package. Next, a soft threshold power $\beta$ was opted for constructing a standard scale-free network. Subsequently, the correlation of genes was calculated using a power function, and an adjacency matrix was thus established. Thereafter, the obtained adjacency matrix underwent remodification into a topological overlap matrix (TOM). Genes then underwent the hierarchical clustering analysis based on dissimilarity (1-TOM), and the dynamic tree cut algorithm was used to seek the modules. Eventually, we applied a height cut-off of 0.25 for the modular dendrogram to merge modules with the most similarities.

\section{Conversion of gene names}

The Ensembl database (http://www.ensembl.org/ index.html) was used to convert the gene names from Ensembl gene stable ID to gene symbol [46].

\section{Construction of the ceRNA network}

Firstly, the overlapping lncRNAs, miRNAs, and mRNAs between those from differential gene expression analysis and WGCNA were selected. Secondly, the genes targeted by $\operatorname{lncRNAs}$ were predicted using LncBase v.2 (http://carolina.imis.athena-innovation.gr/diana_tools/ web/index.php? $r=$ lncbasev2\%2Findex-predicted) [47] and matched with corresponding differentially expressed miRNAs. Thirdly, the miRNAs-targeted genes were predicted using three databases, including TargetScan (http://www.targetscan.org/vert_72/) [48], miRDB (http://mirdb.org/) [49], and miRTarBase (http://mirtarbase.cuhk.edu.cn/php/index.php) [50]. The common predicted genes by these databases were selected and matched with differentially expressed mRNAs. Finally, we constructed and visualized the ceRNA network using Cytoscape version 3.8.0.

\section{Gene functional enrichment analysis}

Using the STRING database, GO and KEGG pathway analyses were conducted to determine the overrepresented biological functions of genes in the ceRNA network (https://www.string-db.org/). GO terms describe the biological functions in three aspects: MF, $\mathrm{CC}$, and BP [51]. KEGG is a database for the identification of high-level functions of a biological system with great utilities [52].

\section{Construction and validation of the prognostic risk model}

The lncRNAs-based survival analysis in the ceRNA network was conducted to indicate the independent prognostic factors in ccRCC. For this purpose, the ccRCC clinical data were acquired from TCGA. Then, samples with a survival time of $<30$ days were excluded. Next, the ccRCC samples were randomly grouped into the training $(\mathrm{n}=257)$ and testing cohorts $(n=256)$ using the "caret" package. Next, LASSO regression and Cox regression analyses were used for the training cohort lncRNAs and a prognostic risk model was developed. Then, the samples in training or testing cohort were divided into the highand low-risk groups based on the median risk score of the training cohort. Subsequently, the Kaplan-Meier survival analysis was used to measure the survival differences between the high- and low-risk groups. The ROC curves were plotted using the "timeROC" [53] package to evaluate the risk model performance by calculating the corresponding AUCs. The PCA and t-SNE analyses were conducted for assessing the distribution pattern of each risk group using "stats" and "Rtsne" packages. Finally, the univariate and multivariate Cox regression analyses were conducted to evaluate if the risk score serves as an independent prognostic factor in comparison to other clinical parameters. 


\section{Analysis of the infiltration levels of immune cells}

The infiltration levels of different immune cells were estimated by the CIBERSORT algorithm [54]. The leukocyte gene expression matrix was employed to identify the 22 immune cell types and their infiltration proportions in ccRCC samples with different risk scores. 1000 permutation count and $\mathrm{p}<0.05$ were set as the threshold.

\section{Analysis of the ICB-related genes}

The differential expression of 47 ICB-related genes between the high- and low-risk samples, and the correlation of ICB-related genes expression and the risk score were evaluated. The results were visualized using the "ggpubr" package. p-value $<0.05$ was considered to be statistically significant.

\section{Abbreviations}

ccRCC: Clear cell renal cell carcinoma; lncRNAs: Long non-coding RNAs; ceRNA: Competing endogenous RNA; TCGA: The cancer genome atlas; WGCNA: Weighted gene co-expression network analysis; GO: Gene ontology; KEGG: Kyoto encyclopedia of genes and genomes; FDR: False discovery rate; FC: Fold change; LASSO: Least absolute shrinkage and selection operator; ICB: Immune checkpoint blockade; RCC: Renal cell carcinoma; ncRNAs: Non-coding RNAs; miRNAs: MicroRNAs; MREs: miRNA response elements; mRNAs: messenger RNAs; TME: Tumor microenvironment; Tregs: Regulatory $\mathrm{T}$ cells; TOM: Topological overlap matrix; 1-TOM: Dissimilarity; DEGs: Differentially expressed genes; MF: Molecular function; CC: Cellular component; BP: Biological process; ROC: Receiver operator characteristic; OS: Overall survival; AUC: Area under the curve; PCA: Principal component analysis; t-SNE: T-distributed stochastic neighbor embedding; MM: Module membership; GS: Gene significance; CTLA4: Cytotoxic T lymphocyte-associated antigen 4; PD-1: Programmed cell death protein 1; PD-L1: PD-1 ligand 1; CAR: Chimeric antigen receptor.

\section{AUTHOR CONTRIBUTIONS}

Peng Zhang and Feng Chen developed the idea. Yuke Zhang and Jiangwen Dai retrieved and analyzed the data. Weifeng Huang, Qingsong Chen, and Wei Chen wrote the manuscript. Qiying He revised the manuscript.

\section{ACKNOWLEDGMENTS}

The authors would like to appreciate the editors and reviewers for their helpful comments on this article.

\section{CONFLICTS OF INTEREST}

The authors declare that they have no conflicts of interest.

\section{FUNDING}

This work was supported by Science \& Technology Department of Sichuan Province (2020YFS0229 and 2020YFS0158).

\section{REFERENCES}

1. Bray F, Ferlay J, Soerjomataram I, Siegel RL, Torre LA, Jemal A. Global cancer statistics 2018: GLOBOCAN estimates of incidence and mortality worldwide for 36 cancers in 185 countries. CA Cancer J Clin. 2018; 68:394-424. https://doi.org/10.3322/caac.21492 PMID:30207593

2. Linehan WM, Ricketts CJ. The Cancer Genome Atlas of renal cell carcinoma: findings and clinical implications. Nat Rev Urol. 2019; 16:539-52. https://doi.org/10.1038/s41585-019-0211-5 PMID:31278395

3. Escudier B, Porta C, Schmidinger M, Rioux-Leclercq N, Bex A, Khoo V, Gruenvald V, Horwich A, and ESMO Guidelines Committee. Renal cell carcinoma: ESMO Clinical Practice Guidelines for diagnosis, treatment and follow-up. Ann Oncol. 2016 (Suppl 5); 27:v58-68. https://doi.org/10.1093/annonc/mdw328 PMID:27664262

4. Cohen HT, McGovern FJ. Renal-cell carcinoma. N Engl J Med. 2005; 353:2477-90.

https://doi.org/10.1056/NEJMra043172

PMID:16339096

5. Hsieh JJ, Purdue MP, Signoretti S, Swanton C, Albiges L, Schmidinger M, Heng DY, Larkin J, Ficarra V. Renal cell carcinoma. Nat Rev Dis Primers. 2017; 3:17009.

https://doi.org/10.1038/nrdp.2017.9 PMID:28276433

6. Ljungberg B, Albiges L, Abu-Ghanem Y, Bensalah K, Dabestani S, Fernández-Pello $S$, Giles RH, Hofmann F, Hora M, Kuczyk MA, Kuusk T, Lam TB, Marconi L, et al. European Association of Urology Guidelines on Renal Cell Carcinoma: The 2019 Update. Eur Urol. 2019; 75:799-810.

https://doi.org/10.1016/i.eururo.2019.02.011 PMID:

7. Sun $Q$, Hao $Q$, Prasanth KV. Nuclear Long Noncoding RNAs: Key Regulators of Gene Expression. Trends Genet. 2018; 34:142-57.

https://doi.org/10.1016/j.tig.2017.11.005 PMID:29249332 
8. Treiber T, Treiber N, Meister G. Publisher Correction: Regulation of microRNA biogenesis and its crosstalk with other cellular pathways. Nat Rev Mol Cell Biol. 2019; 20:321. https://doi.org/10.1038/s41580-0190106-6. PMID:30728477. Erratum for: Nat Rev Mol Cell Biol. 2019; 20:5-20.

9. Salmena L, Poliseno L, Tay Y, Kats L, Pandolfi PP. A ceRNA hypothesis: the Rosetta Stone of a hidden RNA language? Cell. 2011; 146:353-8.

https://doi.org/10.1016/i.cell.2011.07.014

PMID:21802130

10. Barth DA, Slaby O, Klec C, Juracek J, Drula R, Calin GA, Pichler M. Current Concepts of Non-Coding RNAs in the Pathogenesis of Non-Clear Cell Renal Cell Carcinoma. Cancers (Basel). 2019; 11:1580.

https://doi.org/10.3390/cancers11101580

PMID:31627266

11. Calin GA, Croce CM. MicroRNA signatures in human cancers. Nat Rev Cancer. 2006; 6:857-66. https://doi.org/10.1038/nrc1997 PMID:17060945

12. Pan Q, Wang L, Chai S, Zhang H, Li B. The immune infiltration in clear cell Renal Cell Carcinoma and their clinical implications: A study based on TCGA and GEO databases. J Cancer. 2020; 11:3207-15. https://doi.org/10.7150/jca.37285 PMID:32231726

13. Grivennikov SI, Greten FR, Karin M. Immunity, inflammation, and cancer. Cell. 2010; 140:883-99.

https://doi.org/10.1016/j.cell.2010.01.025 PMID:20303878

14. Chevrier S, Levine JH, Zanotelli VR, Silina K, Schulz D, Bacac M, Ries $\mathrm{CH}$, Ailles $\mathrm{L}$, Jewett MA, Moch $\mathrm{H}$, van den Broek M, Beisel C, Stadler MB, et al. An Immune Atlas of Clear Cell Renal Cell Carcinoma. Cell. 2017; 169:736-49.e18.

https://doi.org/10.1016/i.cell.2017.04.016 PMID:28475899

15. Su S, Akbarinejad S, Shahriyari L. Immune classification of clear cell renal cell carcinoma. Sci Rep. 2021; 11:4338.

https://doi.org/10.1038/s41598-021-83767-z

PMID:33619294

16. Jansen CS, Prokhnevska N, Master VA, Sanda MG, Carlisle JW, Bilen MA, Cardenas M, Wilkinson S, Lake R, Sowalsky AG, Valanparambil RM, Hudson WH, McGuire $D$, et al. An intra-tumoral niche maintains and differentiates stem-like CD8 T cells. Nature. 2019; 576:465-70.

https://doi.org/10.1038/s41586-019-1836-5

PMID:31827286

17. Mantovani A, Sica A, Sozzani S, Allavena $P$, Vecchi A, Locati $M$. The chemokine system in diverse forms of macrophage activation and polarization. Trends Immunol. 2004; 25:677-86.

https://doi.org/10.1016/j.it.2004.09.015

PMID:15530839

18. Biswas SK, Mantovani A. Macrophage plasticity and interaction with lymphocyte subsets: cancer as a paradigm. Nat Immunol. 2010; 11:889-96. https://doi.org/10.1038/ni.1937 PMID:20856220

19. Hao NB, Lü MH, Fan YH, Cao YL, Zhang ZR, Yang SM. Macrophages in tumor microenvironments and the progression of tumors. Clin Dev Immunol. 2012; 2012:948098.

https://doi.org/10.1155/2012/948098 PMID:22778768

20. Chen $H$, Pan $Y$, Jin $X$, Chen $G$. Identification of a Four Hypoxia-Associated Long Non-Coding RNA Signature and Establishment of a Nomogram Predicting Prognosis of Clear Cell Renal Cell Carcinoma. Front Oncol. 2021; 11:713346.

https://doi.org/10.3389/fonc.2021.713346 PMID:34386428

21. Liu H, Ye T, Yang X, Lv P, Wu X, Zhou H, Zeng J, Tang K, Ye Z. A Panel of Four-IncRNA Signature as a Potential Biomarker for Predicting Survival in Clear Cell Renal Cell Carcinoma. J Cancer. 2020; 11:4274-83. https://doi.org/10.7150/jca.40421 PMID:32368310

22. Su Y, Zhang T, Tang J, Zhang L, Fan S, Zhou J, Liang C. Construction of Competitive Endogenous RNA Network and Verification of 3-Key LncRNA Signature Associated With Distant Metastasis and Poor Prognosis in Patients With Clear Cell Renal Cell Carcinoma. Front Oncol. 2021; 11:640150.

https://doi.org/10.3389/fonc. 2021.640150 PMID:33869028

23. Yang $\mathrm{H}$, Xiong $\mathrm{X}$, Li $\mathrm{H}$. Development and Interpretation of a Genomic Instability Derived IncRNAs Based Risk Signature as a Predictor of Prognosis for Clear Cell Renal Cell Carcinoma Patients. Front Oncol. 2021; 11:678253.

https://doi.org/10.3389/fonc.2021.678253 PMID:34094983

24. Ponting $\mathrm{CP}$, Oliver $\mathrm{PL}$, Reik $\mathrm{W}$. Evolution and functions of long noncoding RNAs. Cell. 2009; 136:629-41.

https://doi.org/10.1016/j.cell.2009.02.006 PMID:19239885

25. Hauptman N, Glavač D. Long non-coding RNA in cancer. Int J Mol Sci. 2013; 14:4655-69. https://doi.org/10.3390/ijms14034655 PMID:23443164

26. Zhai W, Sun Y, Guo C, Hu G, Wang M, Zheng J, Lin W, Huang $Q$, Li G, Zheng J, Chang C. LncRNA-SARCC suppresses renal cell carcinoma (RCC) progression via 
altering the androgen receptor(AR)/miRNA-143-3p signals. Cell Death Differ. 2017; 24:1502-17. https://doi.org/10.1038/cdd.2017.74 PMID:28644440

27. Li JK, Chen C, Liu JY, Shi JZ, Liu SP, Liu B, Wu DS, Fang ZY, Bao Y, Jiang MM, Yuan JH, Qu L, Wang LH. Long noncoding RNA MRCCAT1 promotes metastasis of clear cell renal cell carcinoma via inhibiting NPR3 and activating p38-MAPK signaling. Mol Cancer. 2017; 16:111.

https://doi.org/10.1186/s12943-017-0681-0

PMID:28659173

28. Chen N, Zhang G, Fu J, Wu Q. Identification of Key Modules and Hub Genes Involved in Esophageal Squamous Cell Carcinoma Tumorigenesis Using WCGNA. Cancer Control. 2020; 27:1073274820978817. https://doi.org/10.1177/1073274820978817 PMID:33345608

29. Zhao W, Langfelder P, Fuller T, Dong J, Li A, Hovarth S. Weighted gene coexpression network analysis: state of the art. J Biopharm Stat. 2010; 20:281-300. https://doi.org/10.1080/10543400903572753 PMID:20309759

30. Moding EJ, Kastan MB, Kirsch DG. Strategies for optimizing the response of cancer and normal tissues to radiation. Nat Rev Drug Discov. 2013; 12:526-42. https://doi.org/10.1038/nrd4003 PMID:23812271

31. Hanahan D, Weinberg RA. Hallmarks of cancer: the next generation. Cell. 2011; 144:646-74.

https://doi.org/10.1016/j.cell.2011.02.013

PMID:21376230

32. Riley RS, June $\mathrm{CH}$, Langer R, Mitchell MJ. Delivery technologies for cancer immunotherapy. Nat Rev Drug Discov. 2019; 18:175-96.

https://doi.org/10.1038/s41573-018-0006-z PMID:30622344

33. Huang D, Chen J, Yang L, Ouyang Q, Li J, Lao L, Zhao J, Liu J, Lu Y, Xing Y, Chen F, Su F, Yao $H$, et al. NKILA IncRNA promotes tumor immune evasion by sensitizing T cells to activation-induced cell death. Nat Immunol. 2018; 19:1112-25.

https://doi.org/10.1038/s41590-018-0207-y

PMID:30224822

34. Liu Y, Li X, Zhang C, Zhang H, Huang Y. LINC00973 is involved in cancer immune suppression through positive regulation of Siglec-15 in clear-cell renal cell carcinoma. Cancer Sci. 2020; 111:3693-704.

https://doi.org/10.1111/cas.14611

PMID: $\underline{32780490}$

35. Şenbabaoğlu Y, Gejman RS, Winer AG, Liu M, Van Allen EM, de Velasco G, Miao D, Ostrovnaya I, Drill E, Luna A, Weinhold N, Lee W, Manley BJ, et al. Tumor immune microenvironment characterization in clear cell renal cell carcinoma identifies prognostic and immunotherapeutically relevant messenger RNA signatures. Genome Biol. 2016; 17:231.

https://doi.org/10.1186/s13059-016-1092-z

PMID:27855702

36. Bi K, He MX, Bakouny Z, Kanodia A, Napolitano S, Wu J, Grimaldi G, Braun DA, Cuoco MS, Mayorga A, DelloStritto L, Bouchard G, Steinharter J, et al. Tumor and immune reprogramming during immunotherapy in advanced renal cell carcinoma. Cancer Cell. 2021; 39:649-61.e5.

https://doi.org/10.1016/i.ccell.2021.02.015 PMID:33711272

37. Xu T, Gao S, Ruan H, Liu J, Liu Y, Liu D, Tong J, Shi J, Yang $\mathrm{H}$, Chen $\mathrm{K}$, Zhang X. METTL14 Acts as a Potential Regulator of Tumor Immune and Progression in Clear Cell Renal Cell Carcinoma. Front Genet. 2021; 12:609174.

https://doi.org/10.3389/fgene.2021.609174 PMID:34122497

38. Facciabene A, Motz GT, Coukos G. T-regulatory cells: key players in tumor immune escape and angiogenesis. Cancer Res. 2012; 72:2162-71. https://doi.org/10.1158/0008-5472.CAN-11-3687 PMID:22549946

39. Kuokkanen E, Šuštar V, Mattila PK. Molecular control of $B$ cell activation and immunological synapse formation. Traffic. 2015; 16:311-26. https://doi.org/10.1111/tra.12257 PMID:25639463

40. Li S, Huang C, Hu G, Ma J, Chen Y, Zhang J, Huang Y, Zheng J, Xue W, Xu Y, Zhai W. Tumor-educated B cells promote renal cancer metastasis via inducing the IL$1 \beta /$ HIF-2 $\alpha /$ Notch1 signals. Cell Death Dis. 2020; 11:163.

https://doi.org/10.1038/s41419-020-2355-x PMID:32123166

41. Cherdantseva TM, Bobrov IP, Avdalyan AM, Klimachev VV, Kazartsev AV, Kryuchkova NG, Klimachev IV, Myadelets MN, Lepilov AV, Lushnikova EL, Molodykh OP. Mast Cells in Renal Cancer: Clinical Morphological Correlations and Prognosis. Bull Exp Biol Med. 2017; 163:801-4.

https://doi.org/10.1007/s10517-017-3907-7 PMID:29063337

42. Nakanishi H, Miyata Y, Mochizuki Y, Yasuda T, Nakamura Y, Araki K, Sagara Y, Matsuo T, Ohba K, Sakai $\mathrm{H}$. Pathological significance and prognostic roles of densities of CD57+ cells, CD68+ cells, and mast cells, and their ratios in clear cell renal cell carcinoma. Hum Pathol. 2018; 79:102-8. https://doi.org/10.1016/i.humpath.2018.05.007 PMID:29787818 
43. Xiong $\mathrm{Y}$, Wang $\mathrm{Z}$, Zhou $\mathrm{Q}$, Zeng $\mathrm{H}$, Zhang $\mathrm{H}$, Liu $\mathrm{Z}$, Huang Q, Wang J, Chang Y, Xia Y, Wang Y, Liu L, Zhu Y, et al. Identification and validation of dichotomous immune subtypes based on intratumoral immune cells infiltration in clear cell renal cell carcinoma patients. J Immunother Cancer. 2020; 8:e000447. https://doi.org/10.1136/jitc-2019-000447 PMID:32217761

44. Robinson MD, McCarthy DJ, Smyth GK. edgeR: a Bioconductor package for differential expression analysis of digital gene expression data. Bioinformatics. 2010; 26:139-40.

https://doi.org/10.1093/bioinformatics/btp616 PMID:19910308

45. Zhang B, Horvath S. A general framework for weighted gene co-expression network analysis. Stat Appl Genet Mol Biol. 2005; 4:Article17. https://doi.org/10.2202/1544-6115.1128 PMID:16646834

46. Aken BL, Ayling S, Barrell D, Clarke L, Curwen V, Fairley S, Fernandez Banet J, Billis K, García Girón C, Hourlier T, Howe K, Kähäri A, Kokocinski $F$, et al. The Ensembl gene annotation system. Database (Oxford). 2016; 2016:baw093.

https://doi.org/10.1093/database/baw093 PMID:27337980

47. Paraskevopoulou MD, Vlachos IS, Karagkouni D, Georgakilas G, Kanellos I, Vergoulis T, Zagganas K, Tsanakas P, Floros E, Dalamagas T, Hatzigeorgiou AG. DIANA-LncBase $\mathrm{v} 2$ : indexing microRNA targets on non-coding transcripts. Nucleic Acids Res. 2016; 44:D231-8.

https://doi.org/10.1093/nar/gkv1270

PMID:26612864

48. Lewis BP, Burge CB, Bartel DP. Conserved seed pairing, often flanked by adenosines, indicates that thousands of human genes are microRNA targets. Cell. 2005; 120:15-20. https://doi.org/10.1016/i.cell.2004.12.035

PMID:15652477

49. Chen $Y$, Wang $X$. miRDB: an online database for prediction of functional microRNA targets. Nucleic Acids Res. 2020; 48:D127-31. https://doi.org/10.1093/nar/gkz757 PMID:31504780

50. Huang HY, Lin YC, Li J, Huang KY, Shrestha S, Hong HC, Tang Y, Chen YG, Jin CN, Yu Y, Xu JT, Li YM, Cai XX, et al. miRTarBase 2020: updates to the experimentally validated microRNA-target interaction database. Nucleic Acids Res. 2020; 48:D148-54. https://doi.org/10.1093/nar/gkz896 PMID:31647101

51. The Gene Ontology Consortium. Expansion of the Gene Ontology knowledgebase and resources. Nucleic Acids Res. 2017; 45:D331-8. https://doi.org/10.1093/nar/gkw1108 PMID:27899567

52. Kanehisa $M$, Furumichi $M$, Sato $Y$, Ishiguro-Watanabe M, Tanabe M. KEGG: integrating viruses and cellular organisms. Nucleic Acids Res. 2021; 49:D545-51. https://doi.org/10.1093/nar/gkaa970 PMID:33125081

53. Blanche $P$, Dartigues JF, Jacqmin-Gadda $H$. Estimating and comparing time-dependent areas under receiver operating characteristic curves for censored event times with competing risks. Stat Med. 2013; 32:5381-97.

https://doi.org/10.1002/sim.5958

PMID:24027076

54. Newman AM, Liu CL, Green MR, Gentles AJ, Feng W, Xu Y, Hoang CD, Diehn M, Alizadeh AA. Robust enumeration of cell subsets from tissue expression profiles. Nat Methods. 2015; 12:453-7. https://doi.org/10.1038/nmeth.3337 PMID:25822800 


\section{SUPPLEMENTARY MATERIALS}

\section{Supplementary Figure}

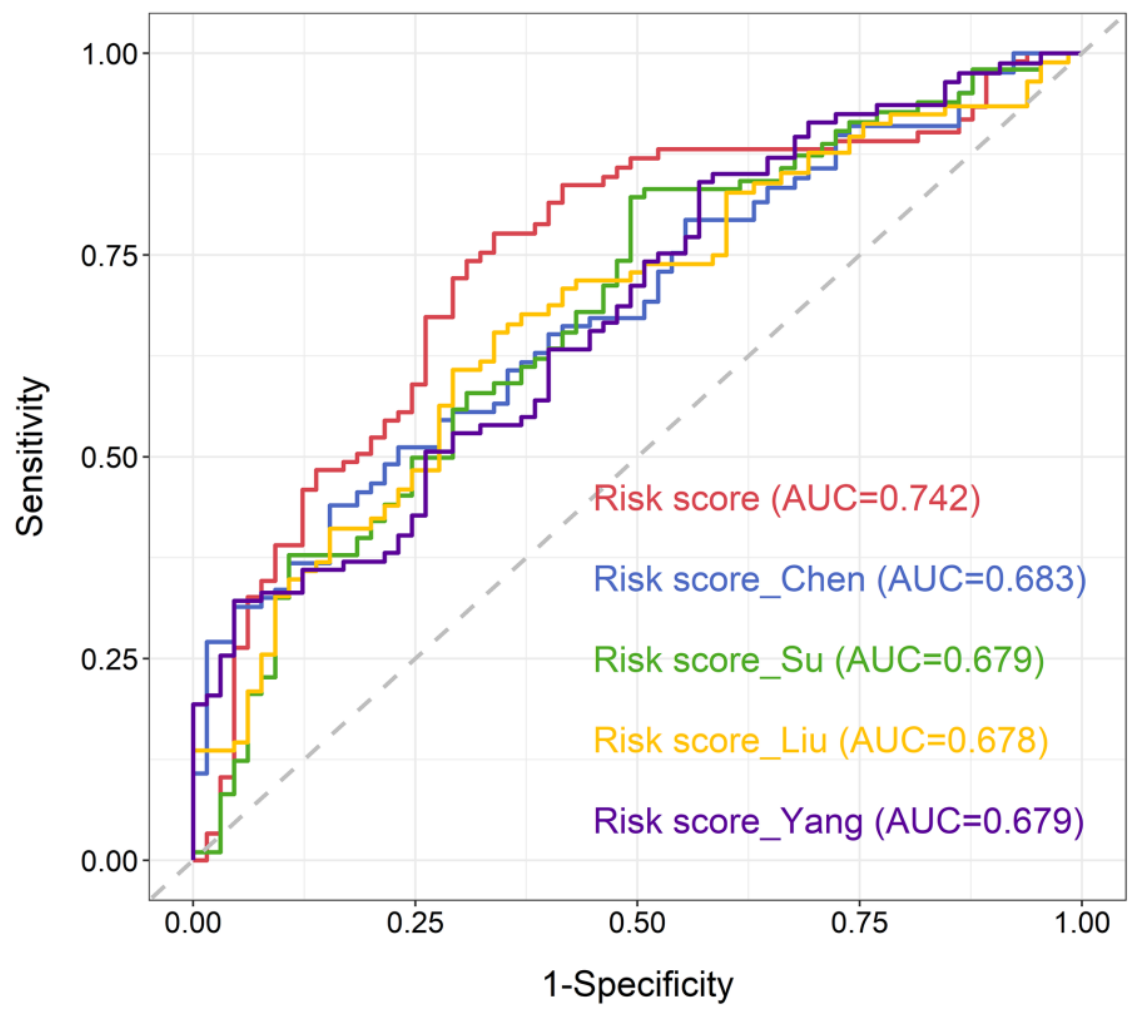

Supplementary Figure 1. ROC curves based on the present and previous prognostic risk models for predicting the 5-year OS in the testing cohort. 


\section{Supplementary Table}

Supplementary Table 1. The correlation between the risk score and ICB-related genes.

\begin{tabular}{|c|c|c|c|}
\hline Risk score & Genes & Correlation & Pvalue \\
\hline Risk score & TNFRSF18 & 0.628998143 & 7.39E-58 \\
\hline Risk score & LAG3 & 0.582342029 & $6.63 \mathrm{E}-48$ \\
\hline Risk score & TNFSF14 & 0.578299472 & $4.07 \mathrm{E}-47$ \\
\hline Risk score & CTLA4 & 0.559690938 & $1.26 \mathrm{E}-43$ \\
\hline Risk score & LAIR1 & 0.554739361 & $9.80 \mathrm{E}-43$ \\
\hline Risk score & TNFRSF8 & 0.539604233 & $4.23 \mathrm{E}-40$ \\
\hline Risk score & TIGIT & 0.53570365 & $1.92 \mathrm{E}-39$ \\
\hline Risk score & LGALS9 & 0.530655091 & $1.33 \mathrm{E}-38$ \\
\hline Risk score & TNFRSF25 & 0.529224992 & $2.28 \mathrm{E}-38$ \\
\hline Risk score & PDCD1 & 0.529215128 & $2.29 \mathrm{E}-38$ \\
\hline Risk score & CD27 & 0.506205589 & $9.80 \mathrm{E}-35$ \\
\hline Risk score & TNFRSF9 & 0.492734013 & $9.83 \mathrm{E}-33$ \\
\hline Risk score & CD80 & 0.487758574 & $5.12 \mathrm{E}-32$ \\
\hline Risk score & ICOS & 0.462756108 & $1.38 \mathrm{E}-28$ \\
\hline Risk score & CD244 & 0.461539911 & $1.99 \mathrm{E}-28$ \\
\hline Risk score & IDO2 & 0.456579955 & $8.79 \mathrm{E}-28$ \\
\hline Risk score & TMIGD2 & 0.433183535 & $7.03 \mathrm{E}-25$ \\
\hline Risk score & CD40LG & 0.4279925 & $2.89 \mathrm{E}-24$ \\
\hline Risk score & CD86 & 0.420920703 & $1.91 \mathrm{E}-23$ \\
\hline Risk score & CD48 & 0.408111515 & $5.21 \mathrm{E}-22$ \\
\hline Risk score & TNFSF4 & 0.406643645 & $7.54 \mathrm{E}-22$ \\
\hline Risk score & CD70 & 0.389525679 & $4.93 \mathrm{E}-20$ \\
\hline Risk score & BTLA & 0.387656784 & $7.67 \mathrm{E}-20$ \\
\hline Risk score & TNFSF9 & 0.38474049 & $1.52 \mathrm{E}-19$ \\
\hline Risk score & CD200R1 & 0.372174827 & $2.68 \mathrm{E}-18$ \\
\hline Risk score & CD160 & 0.36218579 & $2.40 \mathrm{E}-17$ \\
\hline Risk score & $\mathrm{CD} 28$ & 0.359554881 & $4.22 \mathrm{E}-17$ \\
\hline Risk score & CD44 & 0.334013414 & $7.78 \mathrm{E}-15$ \\
\hline Risk score & CD40 & 0.292971982 & $1.30 \mathrm{E}-11$ \\
\hline Risk score & ADORA2A & 0.254870413 & 4.75E-09 \\
\hline Risk score & PDCD1LG2 & 0.248463719 & $1.17 \mathrm{E}-08$ \\
\hline Risk score & TNFRSF4 & 0.233256205 & $9.08 \mathrm{E}-08$ \\
\hline Risk score & TNFRSF14 & 0.22041312 & $4.60 \mathrm{E}-07$ \\
\hline Risk score & VSIR & 0.220110972 & $4.78 \mathrm{E}-07$ \\
\hline Risk score & BTNL2 & 0.183270027 & $2.96 \mathrm{E}-05$ \\
\hline Risk score & IDO1 & 0.166154592 & 0.000156546 \\
\hline Risk score & CD276 & 0.15429199 & 0.000452959 \\
\hline Risk score & TNFSF18 & 0.069039149 & 0.118346459 \\
\hline Risk score & HAVCR2 & 0.062374443 & 0.158339645 \\
\hline Risk score & CD200 & 0.059886251 & 0.175640432 \\
\hline Risk score & HHLA2 & 0.036799909 & 0.405551096 \\
\hline Risk score & CD274 & 0.01448957 & 0.743366739 \\
\hline Risk score & KIR3DL1 & 0.003153622 & 0.943195674 \\
\hline
\end{tabular}


Risk score

Risk score

Risk score

Risk score
ICOSLG

NRP1

TNFSF15

VTCN1
$-0.031925248$

$-0.044113629$

$-0.093830422$

$-0.205208172$
0.470595153

0.318667462

0.03361018

2.78E-06 